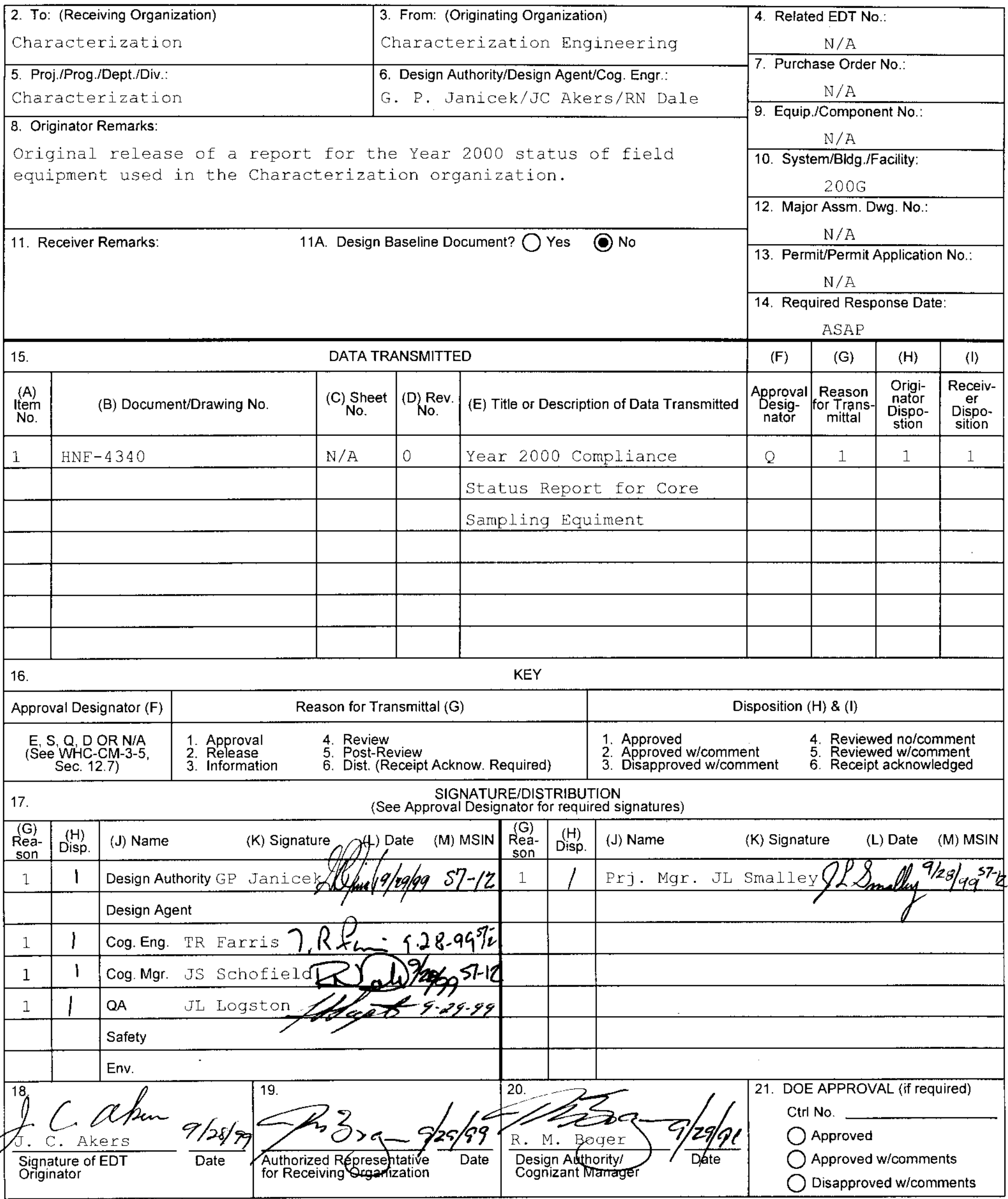




\title{
Year 2000 Compliance Status Report for Core Sampling Equipment
}

\author{
R. M. Boger \\ Lockheed Martin Hanford Co. \\ Richland, WA 99352 \\ U.S. Department of Energy Contract DE-AC06-96RL13200
EDT/ECN: 617972 UC: 2070
Org Code: $83100 \quad$ Charge Code: $102249 /$ B00
B\&R Code: Ew3120074 Total Pages: 23 \\ Key Words: Y2K, core sampling, rotary, exhauster
}

\begin{abstract}
:
Several pieces of equipment that support core sampling activities are "date aware" and could be affected by the roll over to the year 2000. This report documents the testing and remedial efforts for this equipment.
\end{abstract}

TRADEMARK DISCLAIMER. Reference herein to any specific commercial product, process, or service by trade name, trademark, manufacturer, or otherwise, does not necessarily constitute or imply its endorsement, recommendation, or favoring by the United States Government or any agency thereof or its contractors or subcontractors.

Printed in the United States of America. To obtain copies of this document, contact: Document Control Services, P.O. Box 950, Mailstop H6-08, Richland WA 99352, Phone (509) 372-2420; Fax (509) 376-4989.

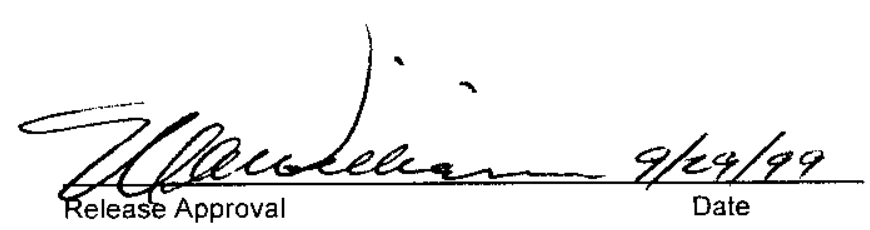

Approved For Public Release 


\section{TABLE OF CONTENTS}

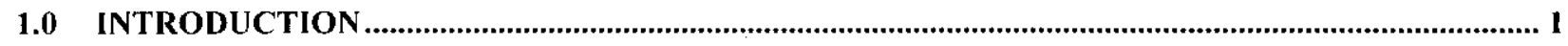

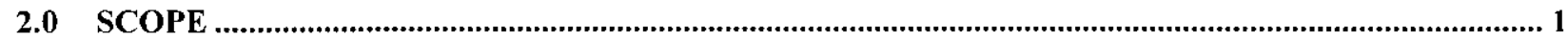

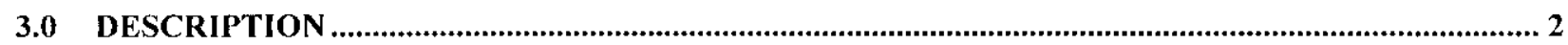

$3.1 \quad$ SYSTEM DESCRIPTION

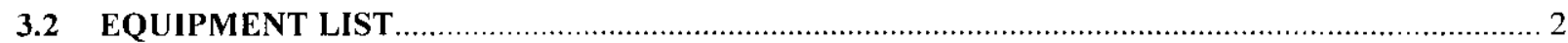

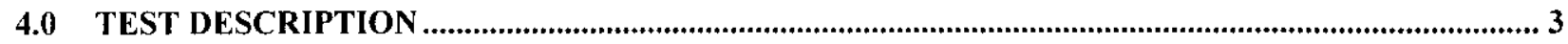

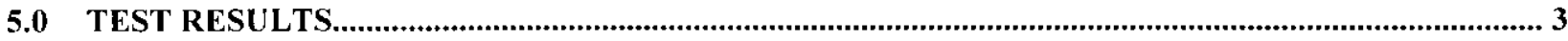

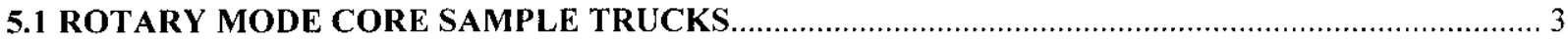

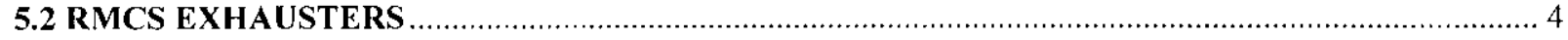

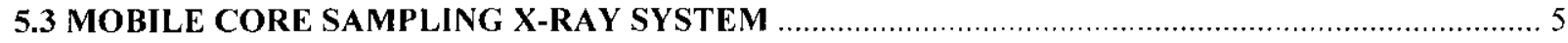

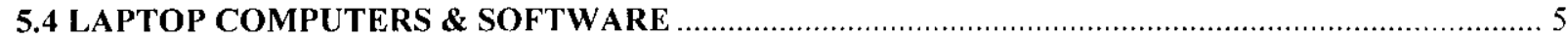

6.0 REMEDIATION …………………........................................................................................................

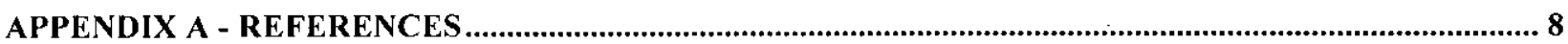

APPENDIX B - MANUFACTURER COMPLIANCE STATEMENTS .............................................................. 9

APPENDIX C- TEST DATA ........................................................................................................................ 10 


\subsection{INTRODUCTION}

There are two Rotary Mode Core Sampling (RMCS) systems that obtain core samples of nuclear waste from various underground tanks. The two primary pieces of equipment in each RMCS system are the RMCS truck and the Exhauster. There are several pieces of RMCS system equipment that are time of day and date aware. This makes those pieces of equipment, and any supporting software, possibly vulnerable to several potential problems related to the approaching Year $2000(\mathrm{Y} 2 \mathrm{~K})$ rollover.

\subsection{EXECUTIVE SUMMARY}

All field equipment, and software, associated with RMCS operations was evaluated or tested for $\mathrm{Y} 2 \mathrm{~K}$ compliance. All field equipment that uses date information passed the $\mathrm{Y} 2 \mathrm{~K}$ tests with the exception of two laptop computers used to support data acquisition and maintenance activities. The two laptops have been replaced and checked for proper operation for their intended functions using existing software. The new laptops, using the same datalogger support software, passed all Y2K tests.

\section{$2.0 \quad$ SCOPE}

This document covers the Year 2000 compliance evaluation and testing of equipment and software that supports core sampling efforts for the Characterization organization(s) in the River Protection Project (RPP). The equipment and software tested for Y2K compliance is listed in section 3.2 EQUIPMENT LIST. A brief summary of that equipment is listed below.

- Rotary Mode Core Sample Trucks (RMCST) 3 \& 4

- Rotary Mode Core Sampling (RMCS) Exhausters B \& C

- Mobile Core Sampling X-ray Systems

- Laptop computers

- Desktop computer (used in stand-alone mode)

- Supporting software.

With the exception of one desktop computer, this report does not include any equipment or software that is used on, or connected to, the Hanford Local Area Network (HLAN). While the desktop computer listed above, and in section 3.2 EQUIPMENT LIST, is connected to HLAN the computer was run in a stand-alone, MicroSoft ${ }^{\mathrm{TM}}$ Disk Operating System (MS-DOS ${ }^{\mathrm{TM}}$ ) mode. This was necessary to run the datalogger manufacturers support software. Any equipment connected to HLAN is the responsibility of Lockheed Martin Services Incorporated (LMSI). Also, this report does not cover any $\mathrm{Y} 2 \mathrm{~K}$ compliance testing or evaluation for the Cone Penetrometer, Light Duty Utility Arm (LDUA), or Long Length Contaminated Equipment (LLCE) systems. 


\subsection{DESCRIPTION}

HNF-4340, REV. 0

PAGE 2 OF 21

\subsection{SYSTEMS DESCRIPTION}

Each RMCST and Exhauster contains a data logger to record various parameters during operation. Also, each Exhauster contains a Beta Particle Continuous Air Monitor (CAM) that monitors the contents of the exhauster outlet. Each of these pieces of equipment is time of day/date aware and therefore possibly vulnerable to several $\mathrm{Y} 2 \mathrm{~K}$ date issues. There are two software packages that support the operation of Beta CAM and RMCST data logger. The software is run on two laptop computers. There is no Safety Class (SC) or Safety Significant (SS) RMCS equipment that is "time of day or date aware". Therefore, none of the equipment or software that was checked for Y2K compliance is Safety Class (SC) or Safety Significant (SS). The equipment and software listed in the next section have been tested for $\mathrm{Y} 2 \mathrm{~K}$ compliance. The test methods, criteria, and results are listed in the following sections.

\section{$3.2 \quad$ EQUIPMENT LIST}

ROTARY MODE CORE SAMPLE TRUCKS

RMCST \# 3 (HO-68K-4600)

RMCST \# 3 Data Logger (LMHC 1997)

Angus Electronics model VGR-16

VideoGraphic Recorder ${ }^{\mathrm{TM}}$ (ANGUS 1994)

Stock \# S23405-2

IBM $^{T M}$ Thinkpad ${ }^{T M}$ Computer

Model \# 755C

Property \# WC49990

PC DOS Version 6.3, Rev. 0

Windows ${ }^{\mathrm{TM}} 3.1$

VGRNet Rev. 1.2 (VGRNET 1994)
RMCST \# 4 (HO-68K-4647)

RMCST \# 4 Data Logger (LMHC 1997)

Angus Electronics model VGR-16

VideoGraphic Recorder ${ }^{\top M}$ (ANGUS 1994)

Stock \# S23405-2

Dell ${ }^{\circledast}$ laptop computer

Model Latitude ${ }^{\circledast}$ CPi D266XT

Property \# WC65877

Windows $^{\mathrm{TM}} 98$

VGRNet Rev. 1.2 (VGRNET 1994)

WINAMS V0.11 (WINAMS 1994)

AST Desktop computer

Model Bravo MS 5200M

Property \# WC63469

Windows ${ }^{\mathrm{TM}} 95$ (run in MS-DOS mode)

VGRNet Rev. 1.2 (VGRNET 1994)

ROTARY MODE CORE SAMPLING EXHAUSTERS

EXHAUSTER B (296-P33)

Eberline AMS-4 (LMHC 1998b)

Beta Particle CAM (AMS4 1994)

Eurotherm/Chessell

Data Logger (CHESSEL 1994)

Model \# 4250G
EXHAUSTER C (296-P34)

Eberline AMS-4 (LMHC 1998a)

Beta Particle CAM (AMS4 1994)

Eurotherm/Chessell

Data Logger (CHESSEL 1994)

Model \# 4250G 
Toshiba Laptop Computer

Model \# T4700CT

Property \# WC51504

MS-DOS ${ }^{\mathrm{TM}}$ version 6.22

Windows $3.1^{\mathrm{TM}}$

WINAMS V0.11 (WINAMS 1994)
Dell ${ }^{\circledast}$ laptop computer

HNF-4340, REV. 0

PAGE 3 OF 21

MOBILE CORE SAMPLE X-RAY SYSTEMS (WHC 1995)

HO-64-5979 HO-64-6042

HO-64-6043 HO-64-6044

\subsection{TEST DESCRIPTION}

Entering the following test cases and then allowing it to run for several minutes tested one piece of equipment, of each type and model.

- $\quad$ September 8, 1999 to September 9, 1999 (tests 9999)

- December 31, 1999 to January 1, 2000 (change of millennium)

- February 28, 2000 to February 29, 2000 (recognition of leap year)

- February 29, 2000 to March 1, 2000 (does not go to February 30 or 31 )

- December 31,2000 to January 1, 2001 (checks for bad leap year calculation)

- February 29, 2001 (should create an error as 2001 is not a leap year)

- December 31, 2000 to January 1, 2001 (shows year 2000 has 366 days)

- Retention of "new" date after equipment power off/on cycle

After a new, future, date and time were entered the unit was de-energized. After several minutes the unit was re-energized and checked to insure that the new date and time settings were retained. The data recorded during testing was processed by VGRNet software and the resulting test data is listed in Appendix C Test Data. The recorded data was processed on a computer that was configured for each of the previously listed test case dates prior to processing the test data. This insured that the VGRNet software had no Y2K problems and can process Y2K data as well. The recorded data from a 3.5" floppy disk and the magnetic tape cartridge was processed during this test to insure both types of media have no Y2K problems.

Configuring the computer to the test cases previously listed and then running the software tested the software packages.

\subsection{TEST RESULTS}

\subsection{ROTARY MODE CORE SAMPLE TRUCKS}

The only piece of equipment on RMCST 3 and 4 that is "aware" of the date and time of day is the Angus Electronics VGR ${ }^{\mathrm{TM}} 16$ data logger listed in Section 3.2 EQUIPMENT LIST. The data logger passed all $\mathrm{Y} 2 \mathrm{~K}$ compliance tests and is fully $\mathrm{Y} 2 \mathrm{~K}$ compliant. The following table details the testing performed. Also, the manufacturer issued a statement of compliance that is included 
in Appendix B MANUFACTURER COMPLIANCE STATEMENT. The data logger displays a two-digit year during operation to conserve display space but records year information in a fourdigit format. The data recorded during testing is listed in Appendix C TEST DATA. The test data was processed using the Angus Electronics VGRNET software package. The testing performed on the VGRNET software is detailed in Section 5.4 LAPTOP COMPUTERS \& SOFTWARE.

\begin{tabular}{|l|c|c|}
\hline \multicolumn{1}{|c|}{ VGR 16 TEST DESCRIPTION } & RESULT & INITIALS/DATE \\
\hline September 8,1999 to September 9,1999 (tests 9999) & PASS & JCA/7-7-98 \\
\hline December 31,1999 to January 1,2000 (change of millennium) & PASS & JCA/7-7-98 \\
\hline February 28, 2000 to February 29,2000 (recognition of leap year) & PASS & JCA/7-7-98 \\
\hline February 29,2000 to March 1,2000 & PASS & JCA $/ 7-7-98$ \\
\hline December 31,2000 to January 1, 2001 (bad leap year calc) & PASS & JCA/7-7-98 \\
\hline February 29, 2001 (should error as 2001 is not a leap year) & PASS & JCA/7-7-98 \\
\hline December 31, 2000 to January 1, 2001 (year 2000 has 366 days) & PASS & JCA/7-7-98 \\
\hline Retention of "new" date after equipment power off/on cycle & PASS & JCA/7-7-98 \\
\hline
\end{tabular}

\subsection{RMCS EXHAUSTERS}

The Exhausters (B \& C) each have two devices that are date "aware". The Eurotherm/Chessell data logger and Eberline AMS-4 CAM listed in Section 3.2 EQUIPMENT LIST are time and date "aware". The data logger passed all Y2K compliance tests and is fully Y2K compliant. The following table details the testing performed. The data loggers on the Exhausters record data on paper, not electronically (on disk or memory card). The data logger is configured to print out a status report once every four hours and prints a brief message when an alarm or out of tolerance condition occurs. Attempts were made to contact he manufacturer concerning a Y2K statement of compliance or non-compliance. The manufacturer provided neither.

\begin{tabular}{|l|c|c|}
\hline \multicolumn{1}{|c|}{ EUROTHERM/CHESSEL TEST DESCRIPTION } & RESULT & INITIALS/DATE \\
\hline September 8, 1999 to September 9, 1999 (tests 9999) & PASS & JCA/7-9-98 \\
\hline December 31,1999 to January 1, 2000 (change of millennium) & PASS & JCA $7-9-98$ \\
\hline February 28, 2000 to February 29, 2000 (recognition of leap year) & PASS & $\mathrm{JCA} / 7-9-98$ \\
\hline February 29, 2000 to March 1, 2000 & PASS & $\mathrm{JCA} / 7-9-98$ \\
\hline December 31,2000 to January 1, 2001 (check bad leap year calc) & PASS & $\mathrm{JCA} / 7-9-98$ \\
\hline February 29, 2001 (should error as 2001 is not a leap year) & PASS & $\mathrm{JCA} / 7-9-98$ \\
\hline December 31, 2000 to January 1, 2001 (year 2000 has 366 days) & PASS & $\mathrm{JCA} / 7-9-98$ \\
\hline Retention of "new" date after equipment power off/on cycle & PASS & $\mathrm{JCA} / 7-9-98$ \\
\hline
\end{tabular}


The Beta Particle CAM was also tested and found to be Y2K compliant. The following table details the testing that was performed.

\begin{tabular}{|l|c|c|}
\hline \multicolumn{1}{|c|}{ EBERLINE AMS-4 TEST DESCRIPTION } & RESULT & INITIALS/DATE \\
\hline September 8, 1999 to September 9, 1999 (tests 9999) & PASS & JCA/7-9-98 \\
\hline December 31,1999 to January 1, 2000 (change of millennium) & PASS & JCA/7-9-98 \\
\hline February 28, 2000 to February 29, 2000 (recognition of leap year) & PASS & JCA/7-9-98 \\
\hline February 29, 2000 to March 1, 2000 & PASS & JCA/7-9-98 \\
\hline December 31, 2000 to January 1, 2001 ( check bad leap year calc) & PASS & JCA/7-9-98 \\
\hline February 29, 2001 (should error as 2001 is not a leap year) & PASS & JCA/7-9-98 \\
\hline December 31, 2000 to January 1, 2001 (year 2000 has 366 days) & PASS & JCA/7-9-98 \\
\hline Retention of "new" date after equipment power off/on cycle & PASS & JCA/7-9-98 \\
\hline
\end{tabular}

\subsection{MOBILE CORE SAMPLING X-RAY SYSTEM}

The x-ray systems listed in Section 3.2 EQUIPMENT LIST have two pieces of equipment on them that are date/time "aware". The videocassette recorder (VCR) and image processor both possess date and time information. The x-ray images that are recorded, and or printed, have the date manually typed in along with other information (tank/riser ID, segment \#, etc.) about the sample being examined. The date/time functions on both pieces of equipment are not used on any of the $\mathrm{x}$-ray systems. Therefore, no $\mathrm{Y} 2 \mathrm{~K}$ compliance testing, or remedial action, was needed or performed on the $\mathrm{x}$-ray systems.

\section{$5.4 \quad$ LAPTOP COMPUTERS \& SOFTWARE}

The IBM laptop computer listed in Section 3.2 EQUIPMENT LIST was tested for Y2K compliance and failed. The details of the test are listed in the following table. This computer was used to support data acquisition in the field from RMCST \# 3 \& 4 .

\begin{tabular}{|c|c|c|}
\hline IBM $^{\top M}$ THINKPAD $\bar{T}^{\top M}$ TEST DESCRIPTION & RESULT & INITIALS/DATE \\
\hline September 8, 1999 to September 9, 1999 (tests 9999 ) & PASS & $\mathrm{JCA} / 5-7-98$ \\
\hline December 31,1999 to January 1,2000 (change of millennium) & FAIL & $\mathrm{JCA} / 5-7-98$ \\
\hline February 28,2000 to February 29,2000 (recognition of leap year) & FAIL & $\mathrm{JCA} / 5-7-98$ \\
\hline February 29,2000 to March 1,2000 & FAIL & $\mathrm{JCA} / 5-7-98$ \\
\hline December 31,2000 to January 1,2001 (bad leap year calc) & FAIL & $\mathrm{JCA} / 5-7-98$ \\
\hline February 29,2001 (should error as 2001 is not a leap year) & FAIL & $\mathrm{JCA} / 5-7-98$ \\
\hline December 31,2000 to January 1, 2001 (year 2000 has 366 days) & FAIL & $\mathrm{JCA} / 5-7-98$ \\
\hline Retention of "new" date after & FAIL & JCA/5-7-98 \\
\hline
\end{tabular}

The Toshiba laptop computer listed in Section 3.2 EQUIPMENT LIST was tested for Y2K compliance and failed. The details of the test are listed in the following table. This computer was used to support the calibration of the CAMs on RMCS Exhausters B and C. 
HNF -4340, REV. 0

PAGE 6 OF 21

\begin{tabular}{|l|l|l|}
\hline \multicolumn{1}{|c|}{ TOSHIBA LAPTOP TEST DESCRIPTION } & RESULT & INITIALS/DATE \\
\hline September 8, 1999 to September 9, 1999 (tests 9999) & PASS & JCA/5-7-98 \\
\hline December 31, 1999 to January 1, 2000 (change of millennium) & FAIL & JCA/5-7-98 \\
\hline February 28, 2000 to February 29,2000 (recognition of leap year) & FAIL & JCA/5-7-98 \\
\hline February 29, 2000 to March 1, 2000 & FAIL & JCA/5-7-98 \\
\hline December 31, 2000 to January 1,2001 (bad leap year calc) & FAIL & JCA/5-7-98 \\
\hline February 29, 2001 (should error as 2001 is not a leap year) & FAIL & JCA/5-7-98 \\
\hline December 31, 2000 to January 1, 2001 (year 2000 has 366 days) & FAIL & JCA/5-7-98 \\
\hline Retention of "new" date after equipment power off/on cycle & FAIL & JCA/5-7-98 \\
\hline
\end{tabular}

The VGRNET software package was tested for Y2K compliance on the AST desktop computer listed in Section 3.2 EQUIPMENT LIST. The computer was run in a stand-alone MS-DOS mode and did not interface with HLAN. The details of the test are listed in the following table. The VGR 16 uses both 3.5" floppy disk and magnetic tape to record data. The tests were conducted on both types of media. The VGRNET software package passed all Y2K compliance tests.

\begin{tabular}{|l|l|l|l|}
\hline \multicolumn{1}{|c|}{ VGRNET TEST DESCRIPTION } & DISK & TAPE & INITIALS/DATE \\
\hline September 8, 1999 to September 9, 1999 (tests 9999) & PASS & PASS & JCA/6-9-98 \\
\hline December 31, 1999 to January 1, 2000 (change of millennium) & PASS & PASS & JCA/6-9-98 \\
\hline February 28, 2000 to February 29, 2000 (recognitjon of leap year) & PASS & PASS & JCA/6-9-98 \\
\hline February 29, 2000 to March 1, 2000 & PASS & PASS & JCA/6-9-98 \\
\hline December 31, 2000 to January 1,2001 (bad leap year calc) & PASS & PASS & JCA/6-9-98 \\
\hline February 29, 2001 (should error as 2001 is not a leap year) & PASS & PASS & JCA/6-9-98 \\
\hline December 31, 2000 to January 1, 2001 (year 2000 has 366 days) & PASS & PASS & JCA/6-9-98 \\
\hline Retention of "new" date after equipment power off/on cycle & PASS & PASS & JCA/6-9-98 \\
\hline
\end{tabular}

The WINAMS software package was tested for Y2K compliance on the AST desktop computer listed in Section 3.2 EQUIPMENT LIST. The computer was run in a stand-alone MS-DOS mode and did not interface with HLAN. The details of the test are listed in the following table. The WINAMS software package passed all Y2K compliance tests.

\begin{tabular}{|l|c|c|}
\hline \multicolumn{1}{|c|}{ WINAMS TEST DESCRIPTION } & RESULT & INITIALS/DATE \\
\hline September 8, 1999 to September 9, 1999 (tests 9999) & PASS & JCA/6-10-98 \\
\hline December 31, 1999 to January 1, 2000 (change of millennium) & PASS & JCA/6-10-98 \\
\hline February 28, 2000 to February 29, 2000 (recognition of leap year) & PASS & JCA/6-10-98 \\
\hline February 29, 2000 to March 1, 2000 & PASS & JCA/6-10-98 \\
\hline December 31, 2000 to January 1, 2001 (bad leap year calc) & PASS & JCA/6-10-98 \\
\hline February 29, 2001 (should error as 2001 is not a leap year) & PASS & JCA/6-10-98 \\
\hline December 31, 2000 to January 1, 2001 (year 2000 has 366 days) & PASS & JCA/6-10-98 \\
\hline Retention of "new" date after equipment power off/on cycle & PASS & JCA/6-10-98 \\
\hline
\end{tabular}




\subsection{REMEDIATION}

Due to the $\mathrm{Y} 2 \mathrm{~K}$ non-compliance of the IBM ${ }^{\mathrm{TM}}$ ThinkPad $^{\mathrm{TM}}$ and Toshiba laptop computers, replacements were required. The Dell ${ }^{\circledR}$ Latitude $^{\circledast}$ laptop computers listed in Section 3.2 EQUIPMENT LIST were purchased to replace the non-compliant laptop computers. Because the two Dell ${ }^{\circledast}$ Latitude ${ }^{\circledast}$ laptop computers are identical only one was tested for Y $2 \mathrm{~K}$ compliance. The following table details the testing performed. The computer passed all tests.

\begin{tabular}{|c|c|c|}
\hline DELL $^{\otimes}$ LATITUDE $^{\circledR}$ TEST DESCRIPTION & RESULT & INITIALS/DATE \\
\hline September 8, 1999 to September 9, 1999 (tests 9999) & PASS & $\mathrm{JCA} / 2-16-99$ \\
\hline December 31,1999 to January 1,2000 (change of millennium) & PASS & $\mathrm{JCA} / 2-16-99$ \\
\hline February 28,2000 to February 29,2000 (recognition of leap year) & PASS & $\mathrm{JCA} / 2-16-99$ \\
\hline February 29,2000 to March 1,2000 & PASS & $\mathrm{JCA} / 2-16-99$ \\
\hline December 31,2000 to January 1,2001 (bad leap year calc) & PASS & JCA/2-16-99 \\
\hline February 29,2001 (should error as 2001 is not a leap year) & PASS & $\mathrm{JCA} / 2-16-99$ \\
\hline December 31,2000 to January 1,2001 (year 2000 has 366 days) & PASS & $\mathrm{JCA} / 2-16-99$ \\
\hline Retention of "new" date after equipment power off/on cycle & PASS & JCA/2-16-99 \\
\hline
\end{tabular}

The VGRNet software was transferred to a Dell ${ }^{\oplus}$ Latitude $^{\circledast}$ laptop computer (WC65877) and tested again for compliance. The following table details the testing. This configuration passed all $\mathrm{Y} 2 \mathrm{~K}$ testing.

\begin{tabular}{|c|c|c|}
\hline VGRNET \& DELL ${ }^{\circledR}$ LATITUDE $^{\circledast}$ TEST DESCRIPTION & RESULT & INITIALS/DATE \\
\hline September 8,1999 to September 9,1999 (tests 9999 ) & PASS & $\mathrm{JCA} / 2-26-99$ \\
\hline December 31,1999 to January 1,2000 (change of millennium) & PASS & $\mathrm{JCA} / 2-26-99$ \\
\hline February 28,2000 to February 29,2000 (recognition of leap year) & PASS & $\mathrm{JCA} / 2-26-99$ \\
\hline February 29,2000 to March 1,2000 & PASS & $\mathrm{JCA} / 2-26-99$ \\
\hline December 31,2000 to January 1,2001 (bad leap year calc) & PASS & $\mathrm{JCA} / 2-26-99$ \\
\hline February 29,2001 (should error as 2001 is not a leap year) & PASS & $\mathrm{JCA} / 2-26-99$ \\
\hline December 31,2000 to January 1,2001 (year 2000 has 366 days) & PASS & $\mathrm{JCA} / 2-26-99$ \\
\hline Retention of "new" date after equipment power off/on cycle & PASS & $\mathrm{JCA} / 2-26-99$ \\
\hline
\end{tabular}

The WINAMS software was transferred to a Dell ${ }^{\oplus}$ Latitude ${ }^{\circledast}$ laptop computer (WC65874) and tested again for compliance. The following table details the testing. This configuration passed all $\mathrm{Y} 2 \mathrm{~K}$ testing.

\begin{tabular}{|c|c|c|}
\hline VGRNET \& DELL ${ }^{\circledR}$ LATITUDE $^{\circledR}$ TEST DESCRIPTION & RESULT & INITIALS/DATE \\
\hline September 8,1999 to September 9,1999 (tests 9999 ) & PASS & JCA/2-25-99 \\
\hline December 31,1999 to January 1,2000 (change of millennium) & PASS & $\mathrm{JCA} / 2-25-99$ \\
\hline February 28,2000 to February 29,2000 (recognition of leap year) & PASS & $\mathrm{JCA} / 2-25-99$ \\
\hline February 29,2000 to March 1,2000 & PASS & $\mathrm{JCA} / 2-25-99$ \\
\hline December 31,2000 to January 1,2001 (bad leap year calc) & PASS & $\mathrm{JCA} / 2-25-99$ \\
\hline February 29,2001 (should error as 2001 is not a leap year) & PASS & $\mathrm{JCA} / 2-25-99$ \\
\hline December 31,2000 to January 1,2001 (year 2000 has 366 days) & PASS & $\mathrm{JCA} / 2-25-99$ \\
\hline Retention of "new" date after & PASS & JCA/2-25-99 \\
\hline
\end{tabular}




\section{APPENDIX A - REFERENCES}

WHC 1995, H-2-79076, MOBILE CORE SAMPLE X-RAY SYSTEMS, Westinghouse Hanford LMHC 1997, H-2-690068, Instrument Enclosure Assembly (RMCST 3 \& 4), Lockheed Martin LMHC 1998a, H-2-829086, Instrumentation and Control Cabinet Assembly (RMCST Exhauster LMHC 1998b, H-2-829125, Instrumentation and Control Cabinet Assembly (RMCST Exhauster WINAMS 1994, WINAMS, AMS-4 Beta Particle Continuous Air Monitor Calibration Software AMS4 1994, AMS4.MAN, AMS-4 Beta Particulate Monitor Technical Manual, Eberline, Santa ANGUS 1994, M902A010, VideoGraphic Recorder TM Operating Instructions, Angus VGRNET 1994, VGRNet Software, Revision I.2, Angus Electronics Company, Speedway, CHESSELL 1994, HA245000, Model 4200/4250M Chart Recorder Installation and Operation 


\section{APPENDIX B - MANUFACTURER COMPLIANCE STATEMENTS}

\section{ANGUS}

A Division of Westronics Inc.

Year 2000 compatibility (Y2K)

$10 / 16 / 97$

Please consider this document as our official statement on $\mathrm{Y} 2 \mathrm{~K}$ compatibility. You may fax or copy this document and file it in your records.

Y2K date transition (transition from 31 Dec 1999 to 1 Jan 2000) is successful in the VGR product operation. The VGR is an Intel microprocessor based product that uses an IEEE date format that uses foux (4) digits for the date. Therefore the transition from 1999 to 2000 will not be a problem for the VGR.

Y2K leap year (transition from 28 Feb 2000 to 29 Feb 2000)

will be a successfully transition. The firmware inside the VGR has been developed with high level development tools that handle this issue.

Brian Mattox, Sr. Application Engineer

Fax $1-800-899-5160$

\section{TEThermo Electron}




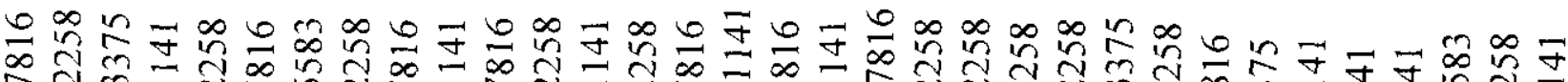
제

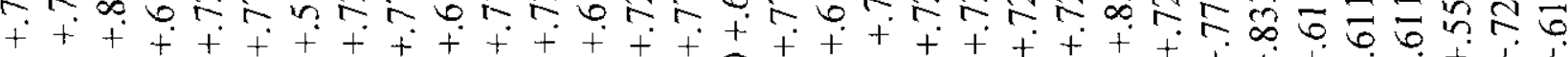

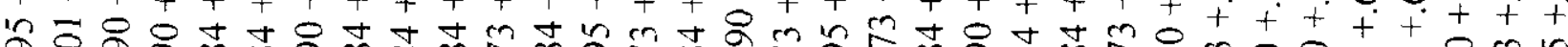

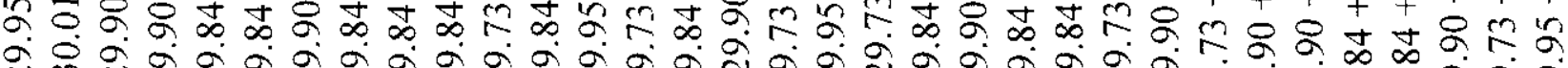
ปิ

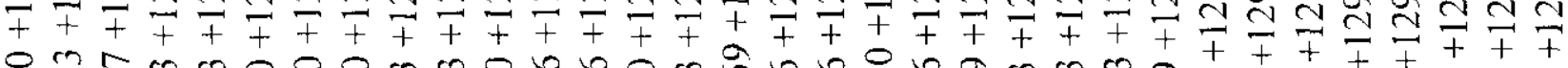

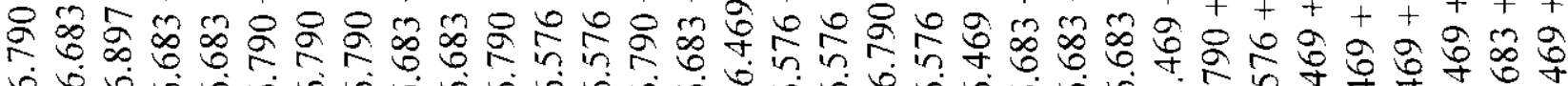

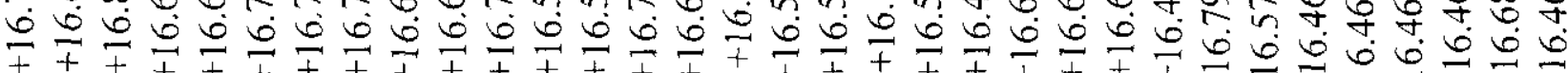
○ 岕岁

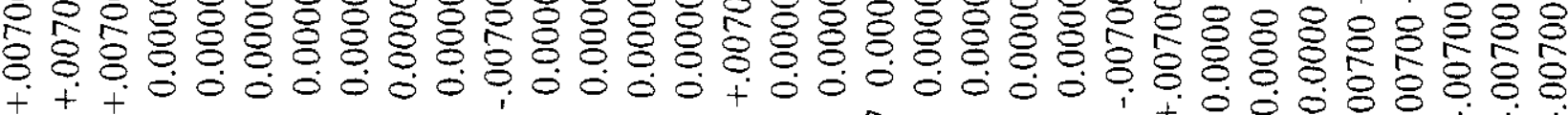

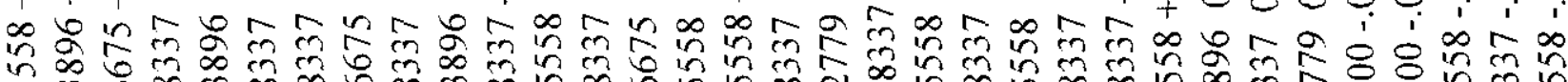

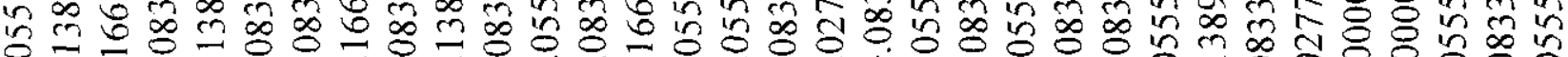
$+++++++++++++++++++++++++0.00000000$

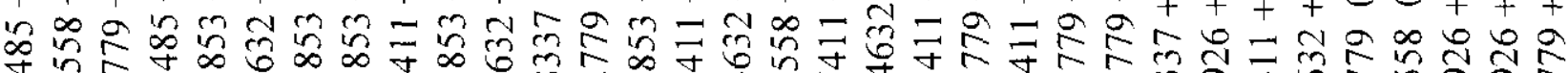

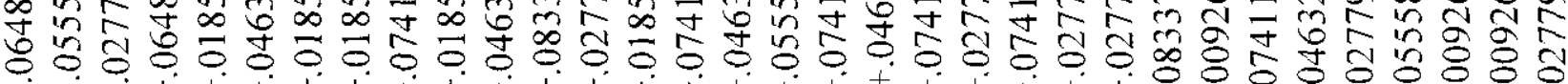
+

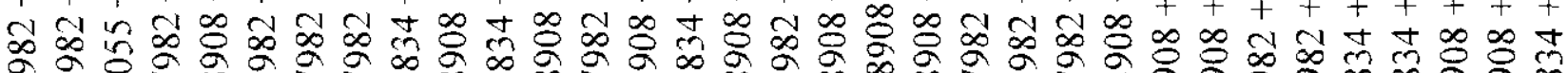

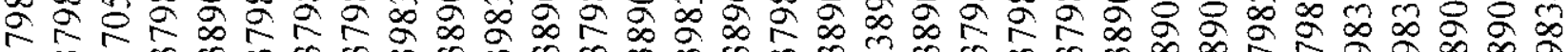

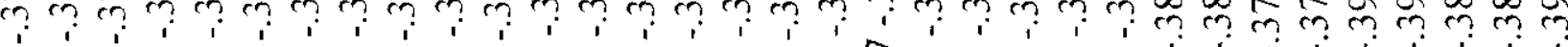

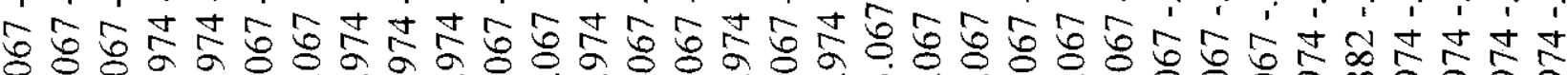

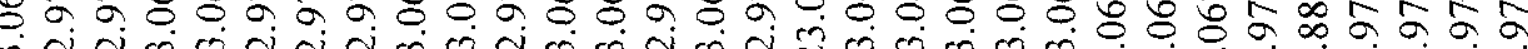

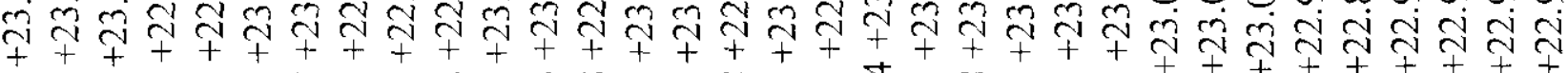

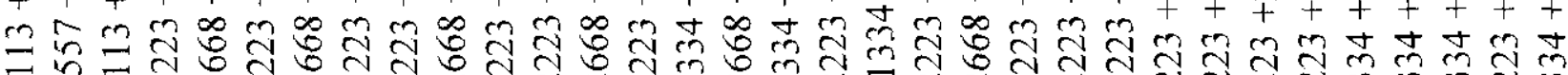

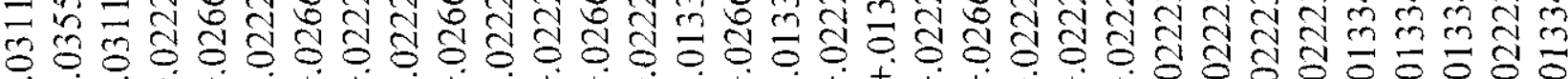

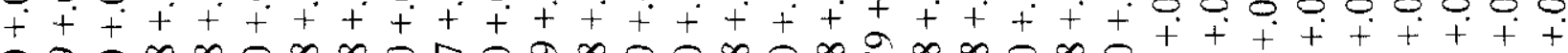

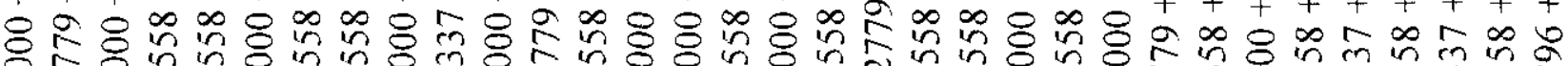
됴유.

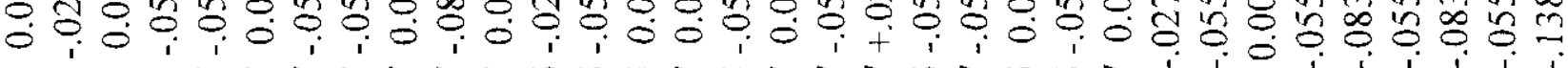

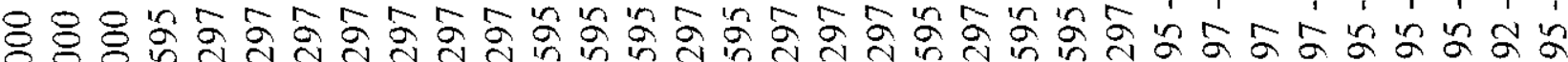
$8 \delta^{\circ}$

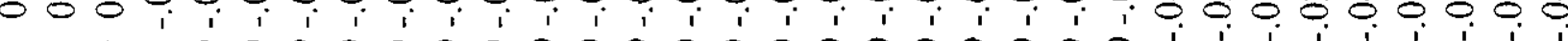

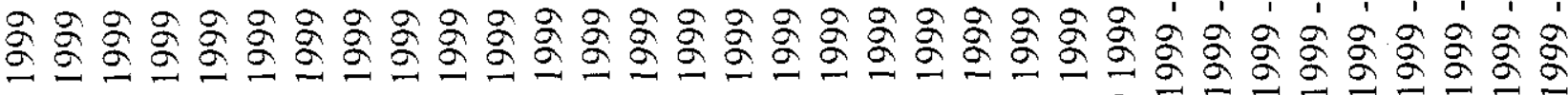

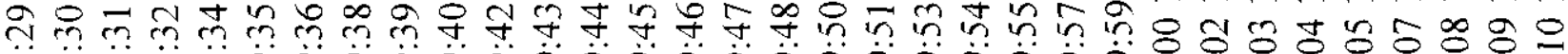

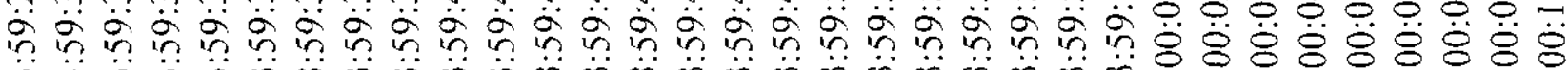

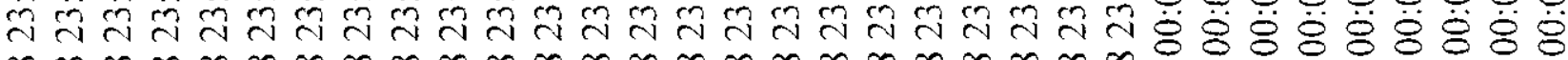

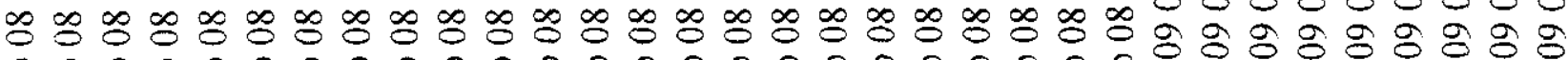

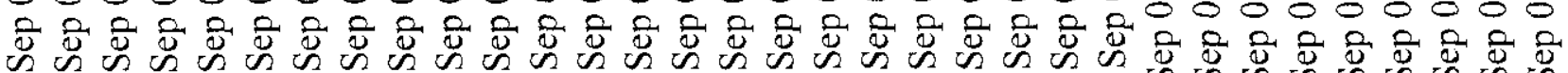

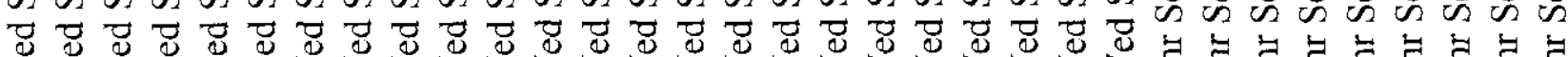

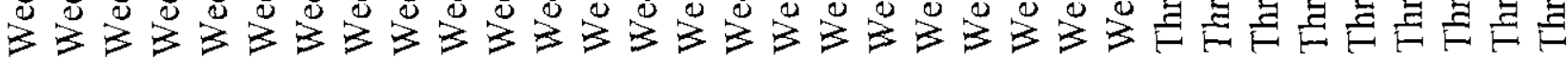




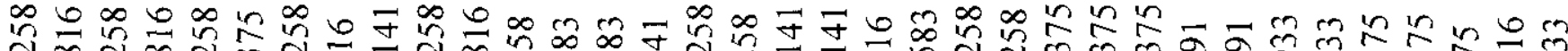

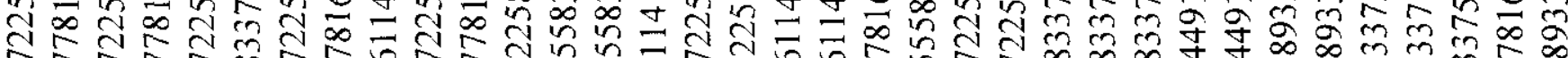

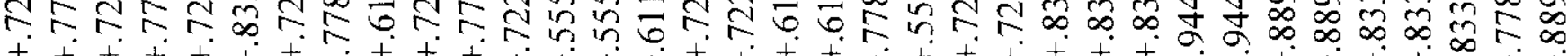

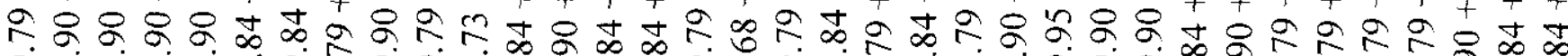

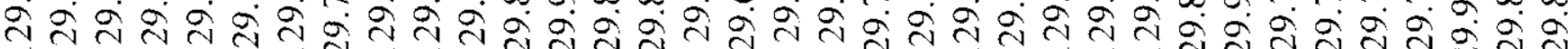

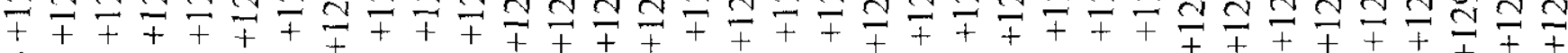

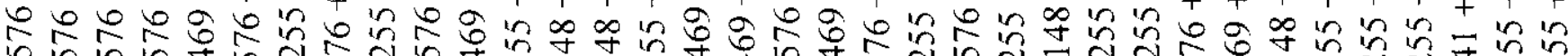

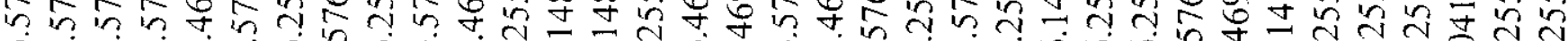

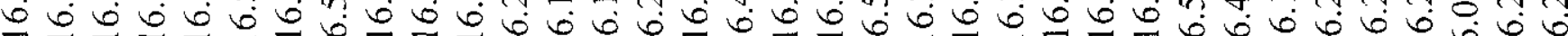
○

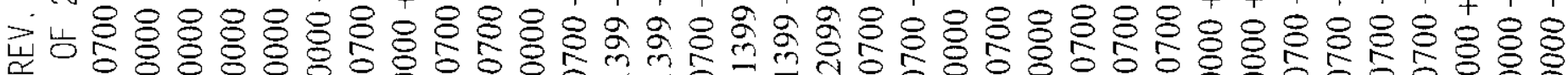

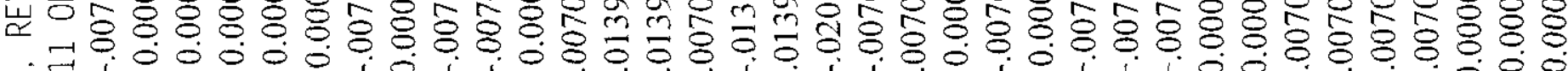

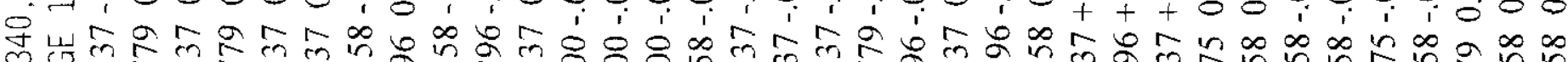

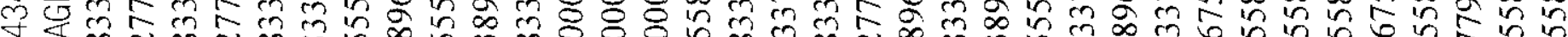

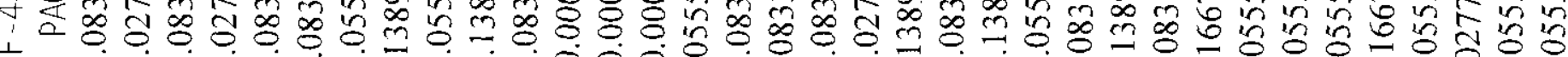

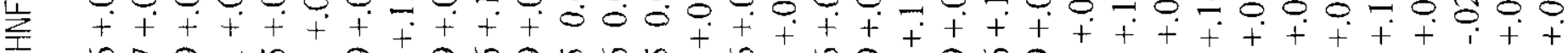
스유 و

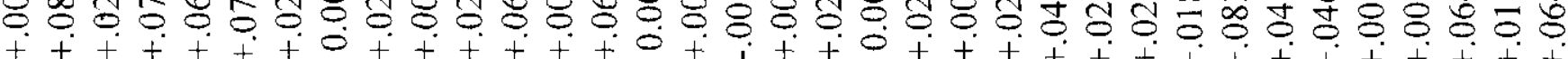

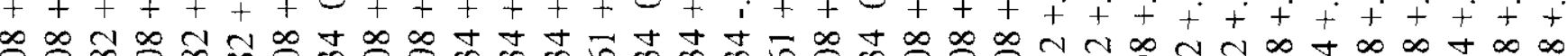
央

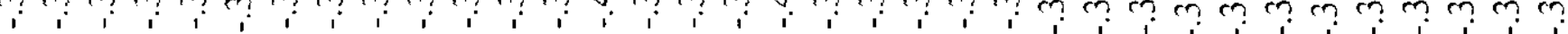

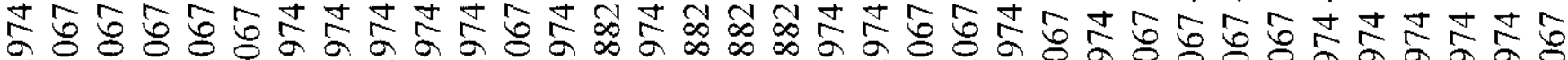
ป่

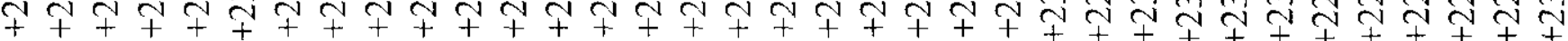
অ

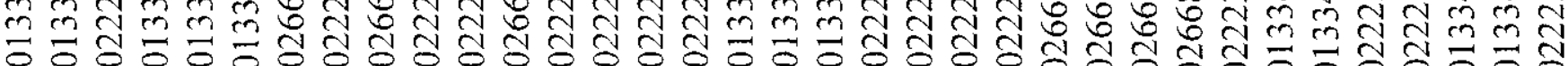

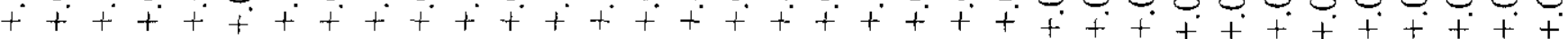

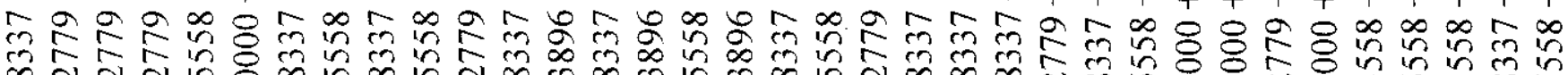

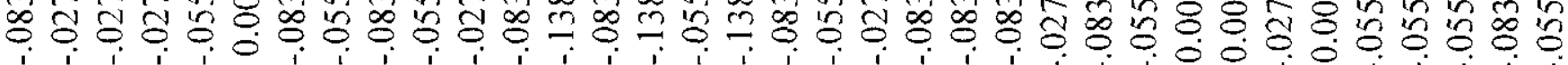

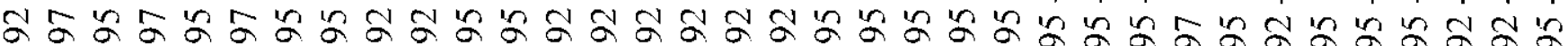

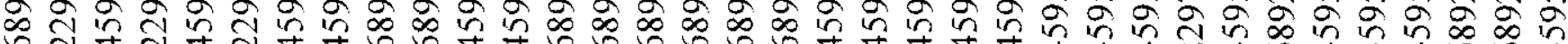

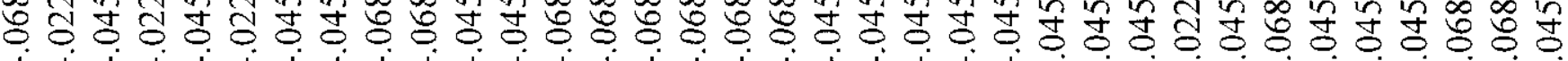

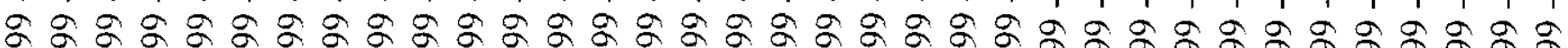

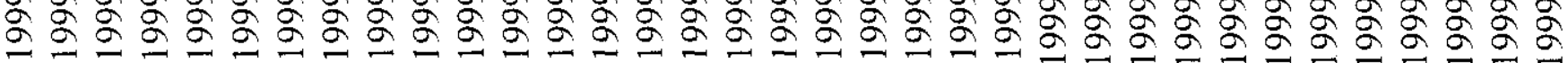

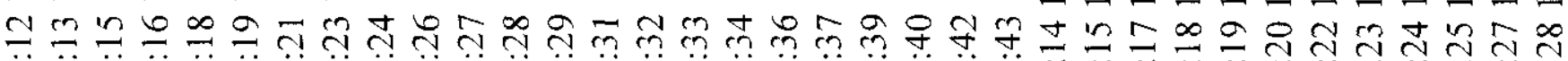

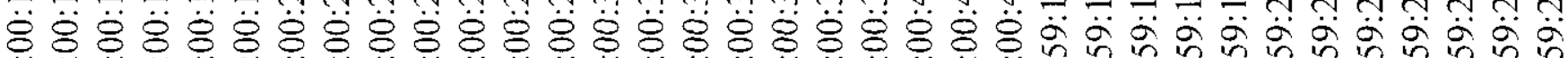

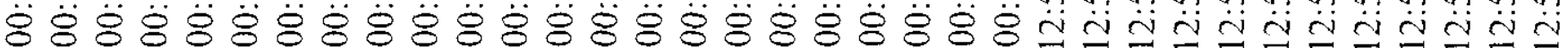
g.

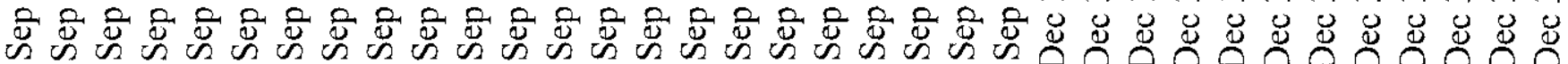

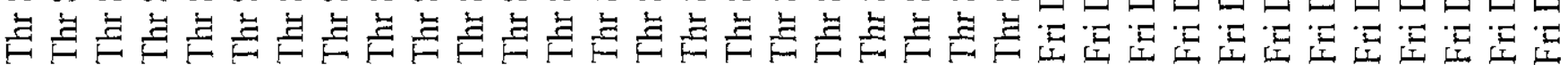




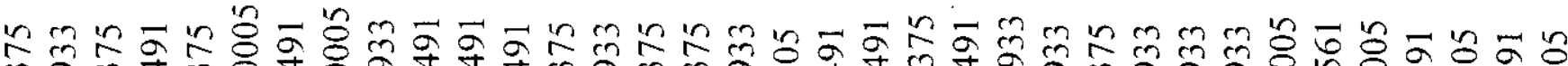

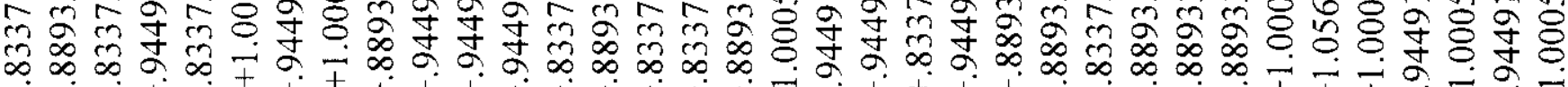

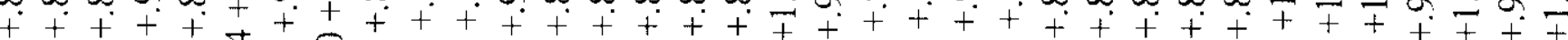
ஓ丈 वें वें बे

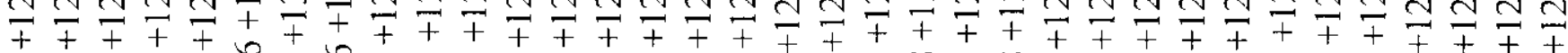

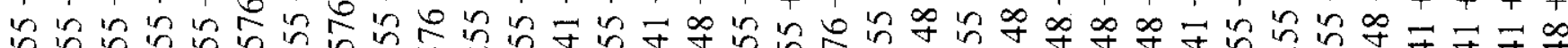
ก้ว

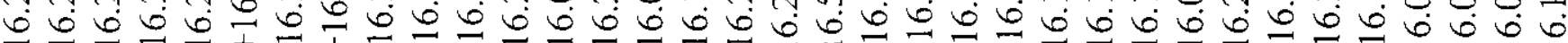

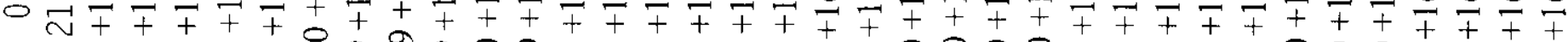

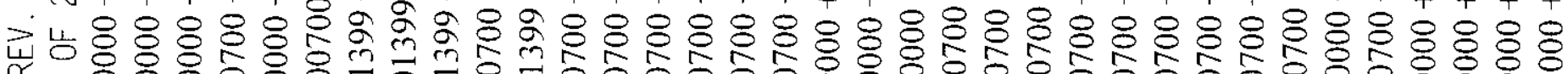

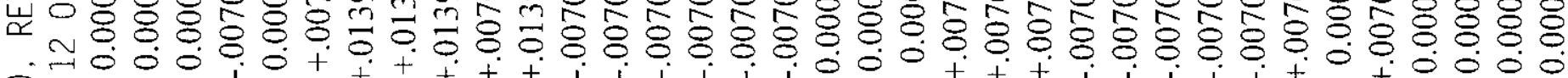

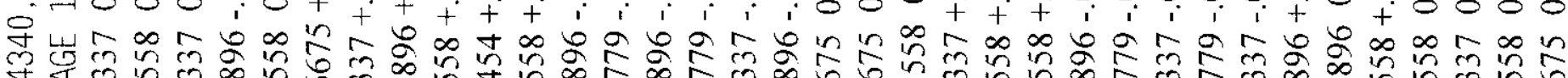

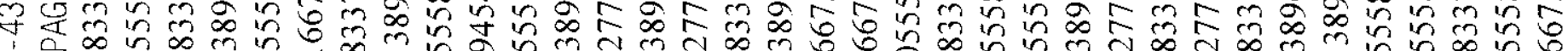

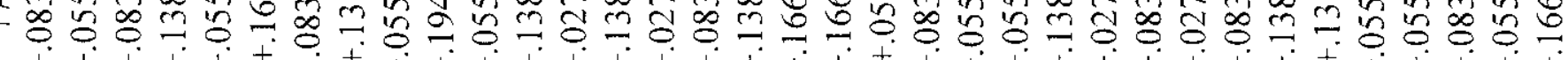

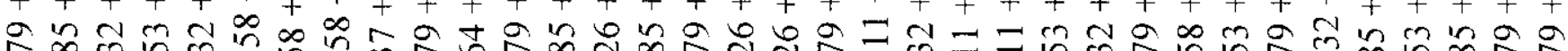

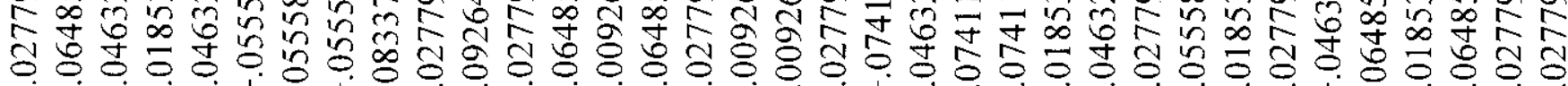
+

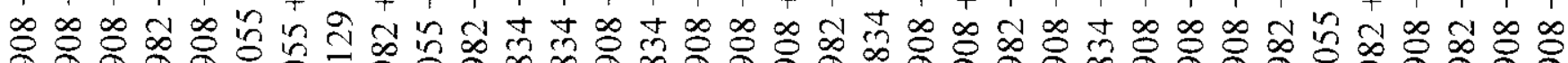

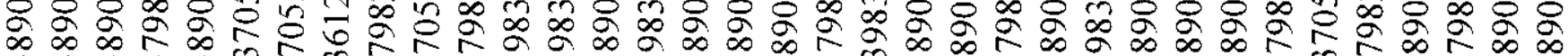

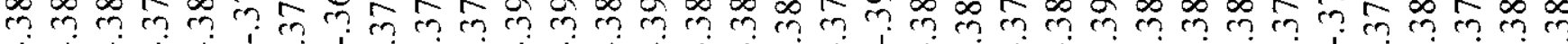

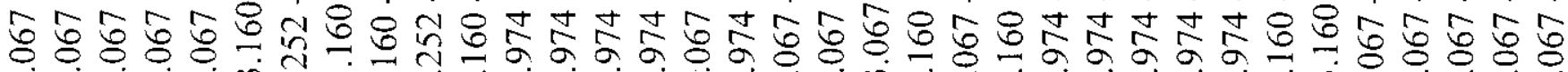


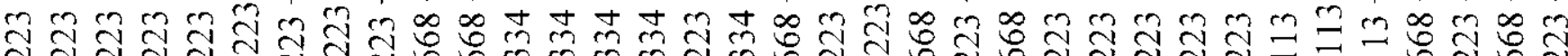

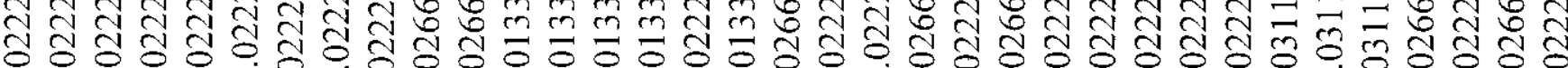
$+++++++++++++++++++++++++++++++++++t+$

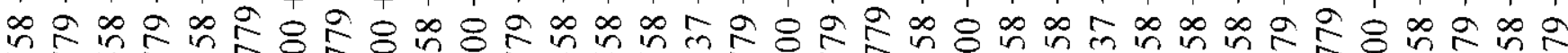

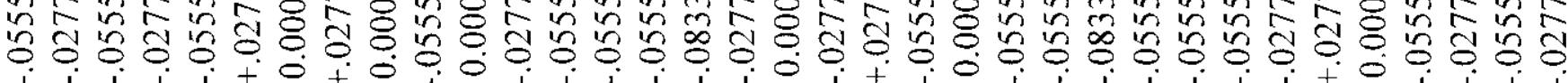

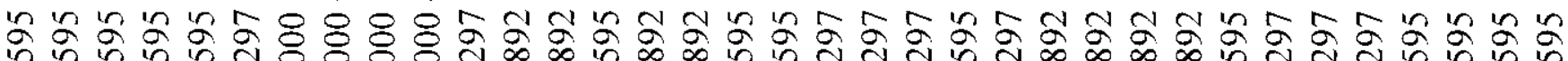

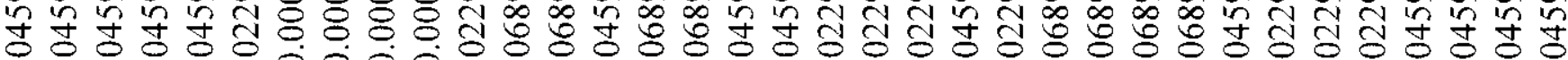

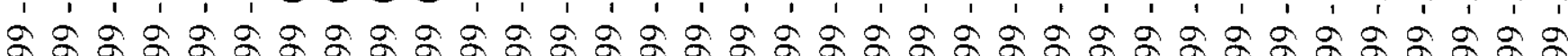
ㅇㅎㅇ 을

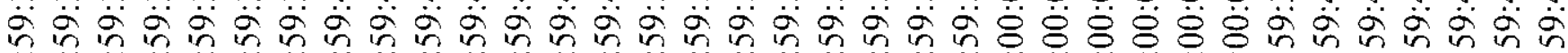

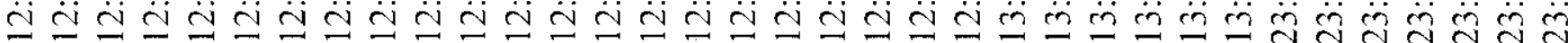

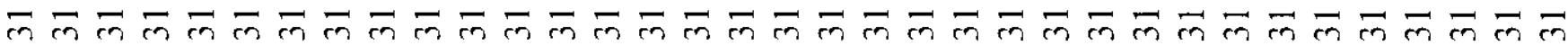

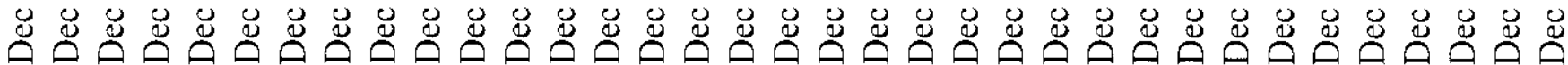

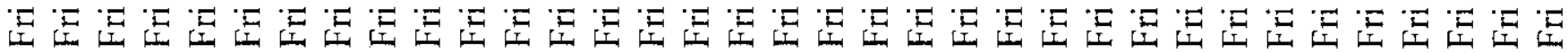




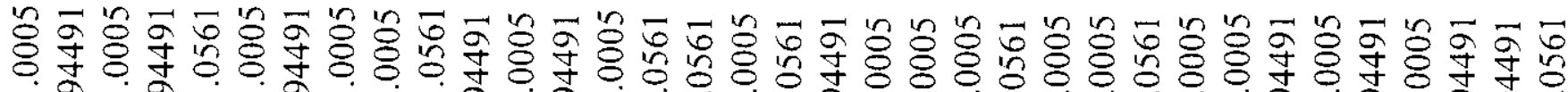

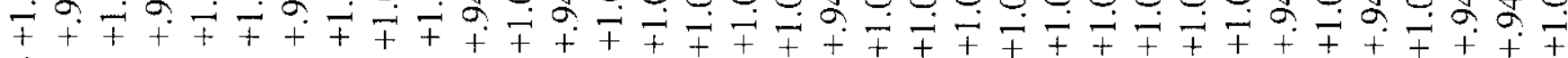
চ

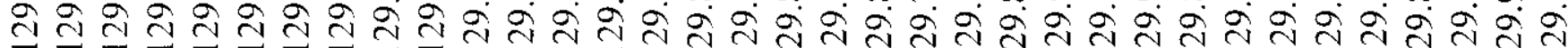

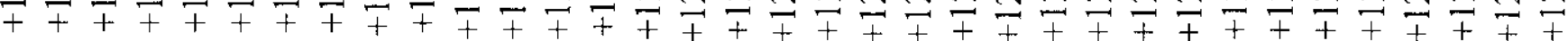

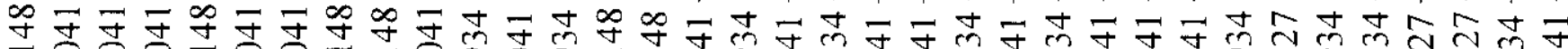
o

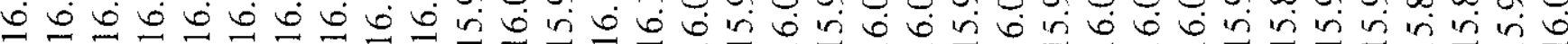

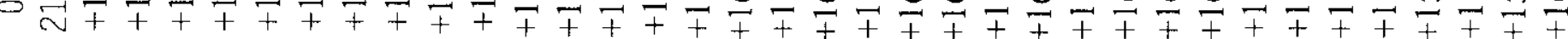
uᄂ

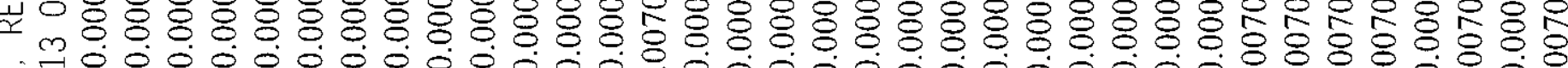
(

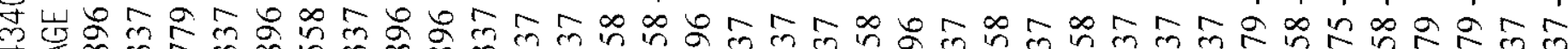

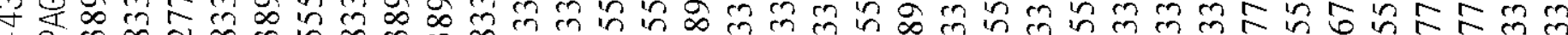

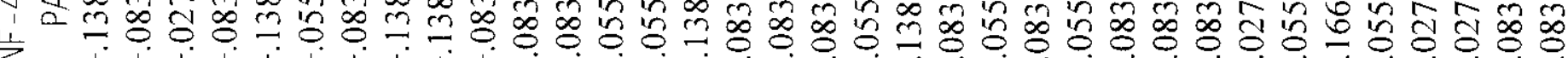

자 $\dot{t}+\dot{+}+\dot{+}+\dot{+}+\dot{+}+\dot{+}+\dot{+}+\dot{+}+\dot{+}+\dot{+}+\dot{+}+\dot{+}+\dot{+}+\dot{+}+\dot{+}+\dot{+}$

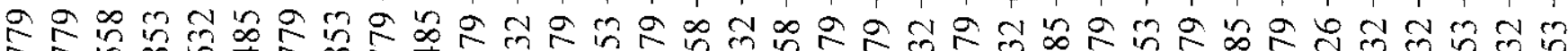
츳

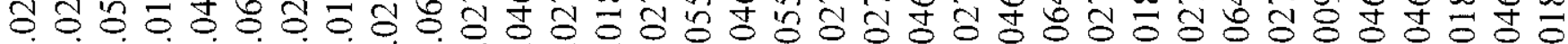

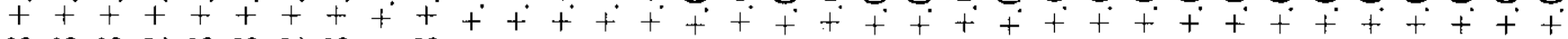
央官

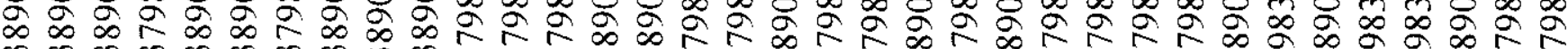

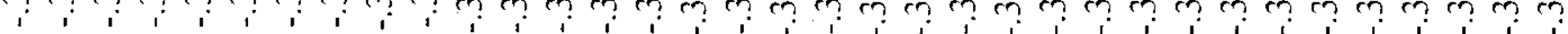

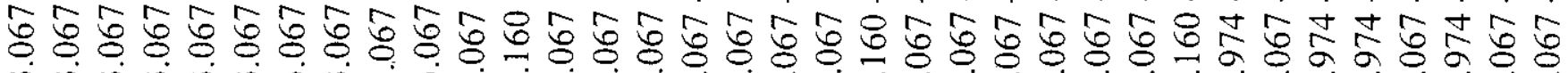

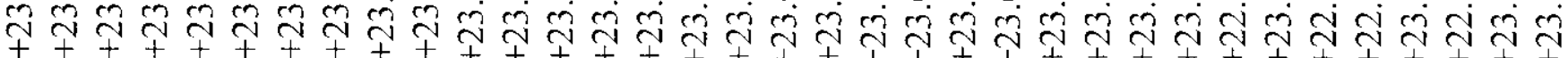

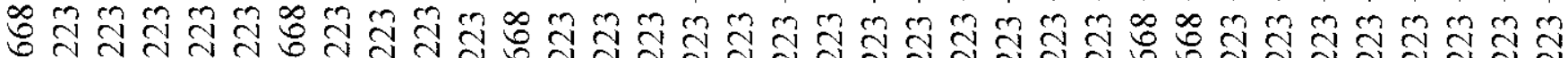

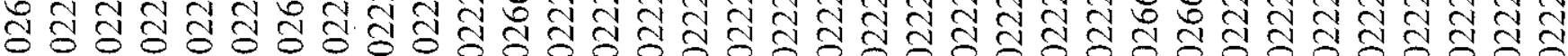
$++++++++++\dot{t}+\dot{+}+\dot{+}+\dot{+}++\dot{+}+\dot{+}+\dot{+}+\dot{+}+\dot{+}+\dot{+}+\dot{0}$

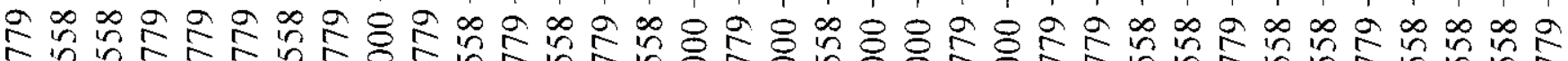

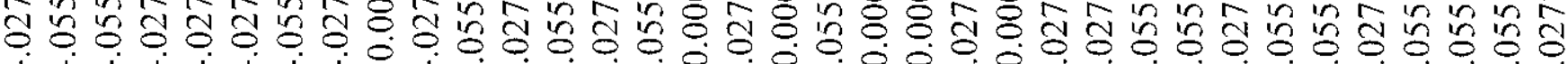

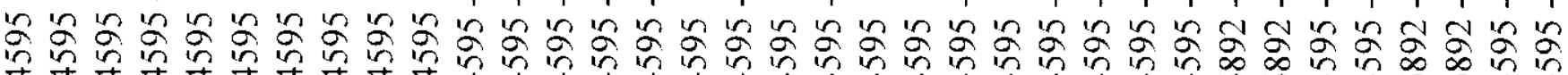

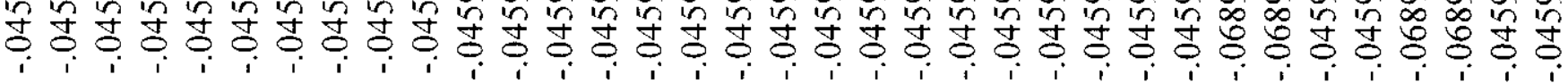

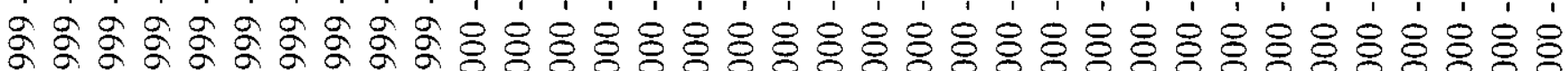

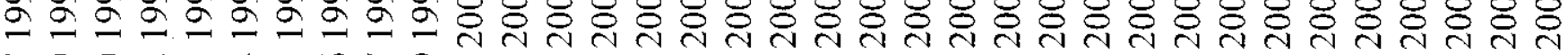

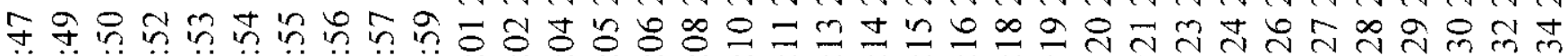
๙人் শึ

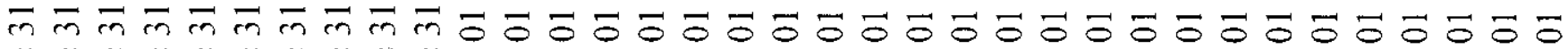

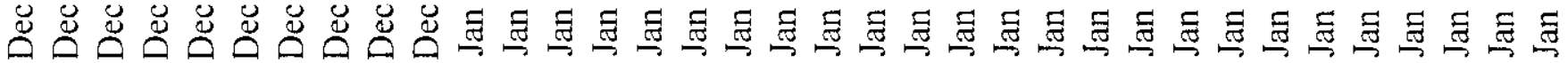

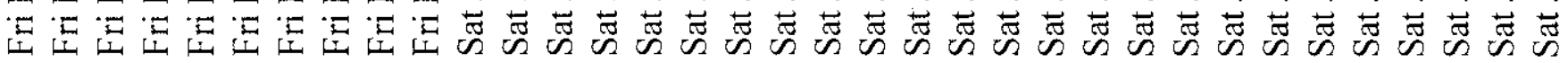




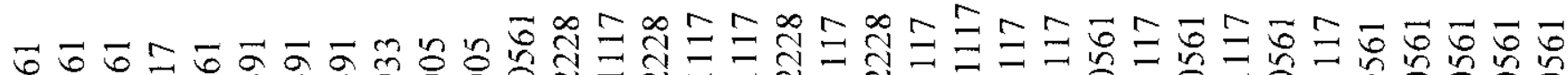

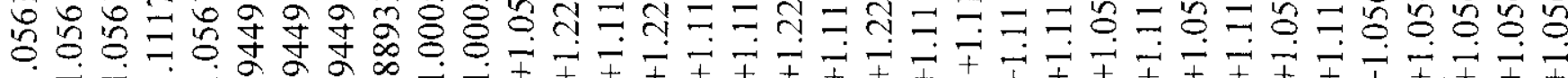

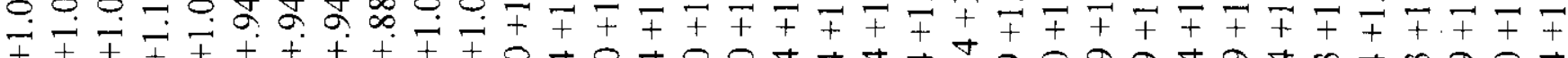

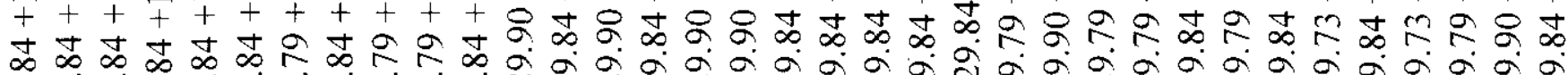
等

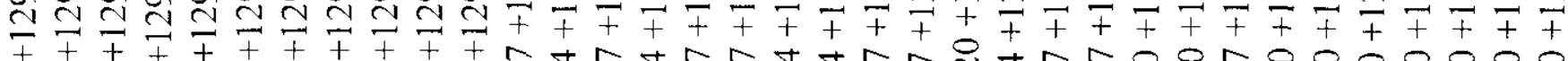

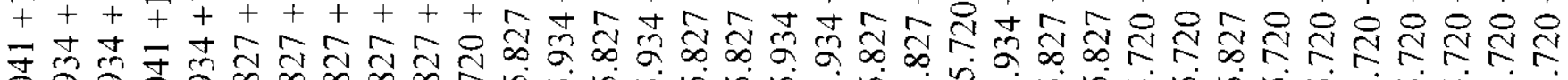
0.000 .

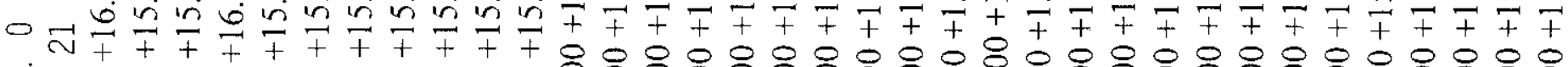

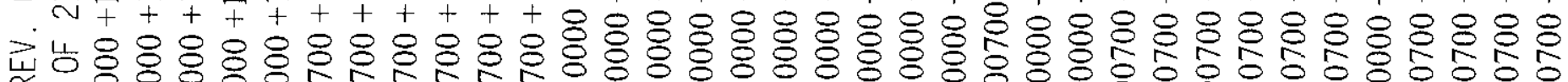

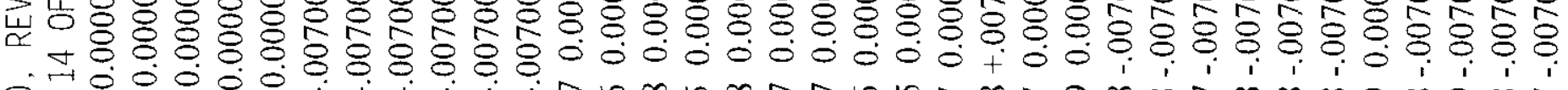
욱

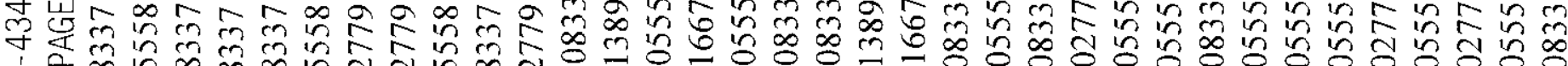

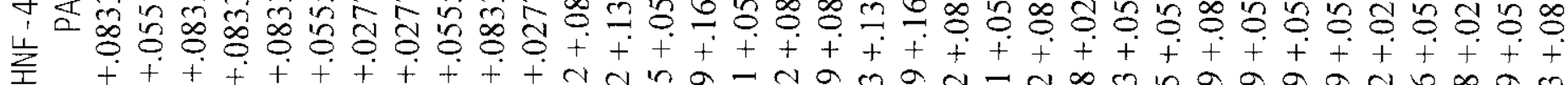

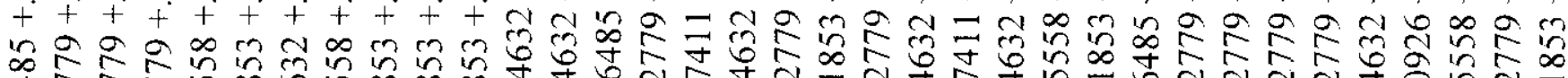

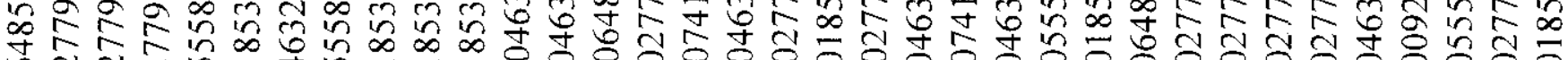
응 $++++++++++\infty)+\infty$

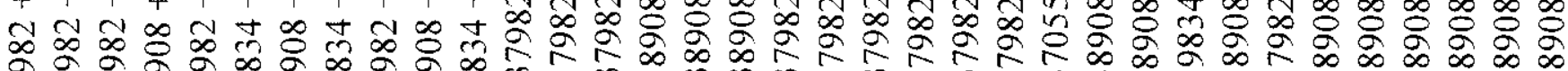
م.

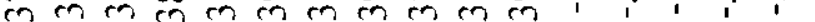

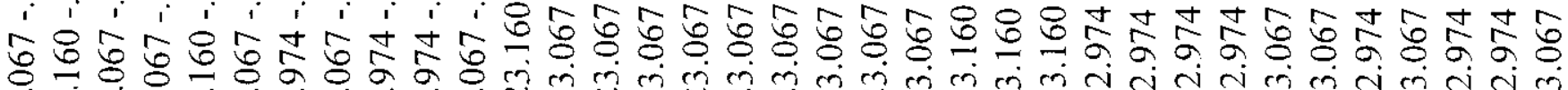

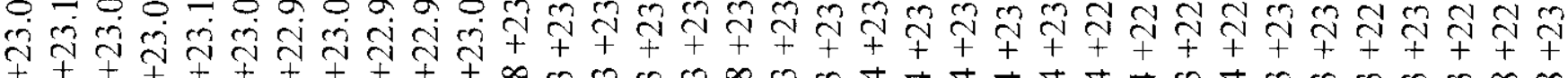
ㄱำ

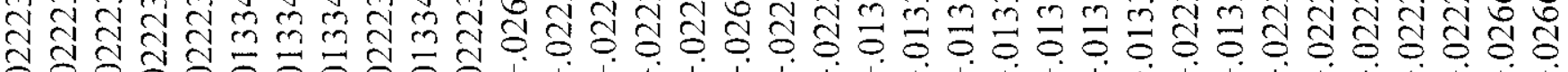

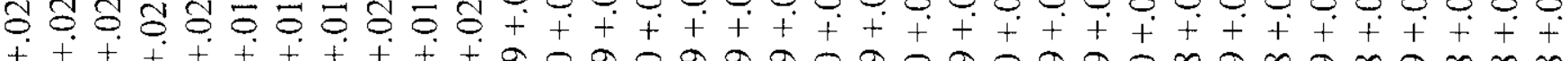

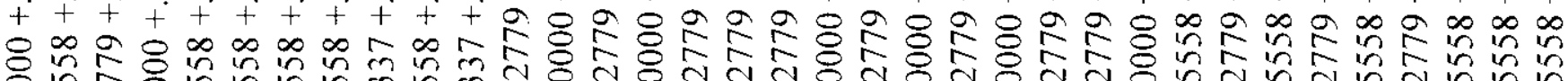

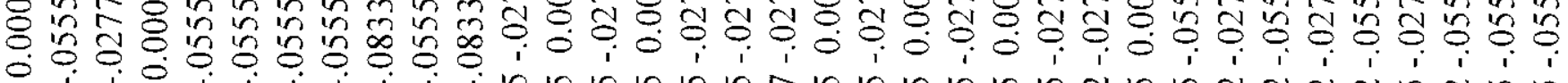

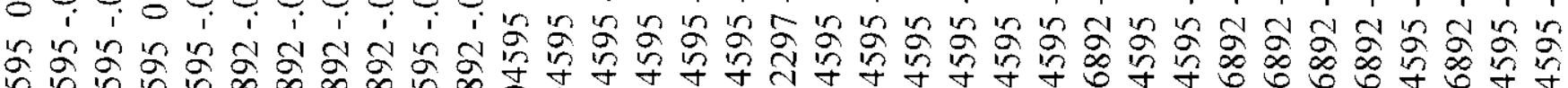

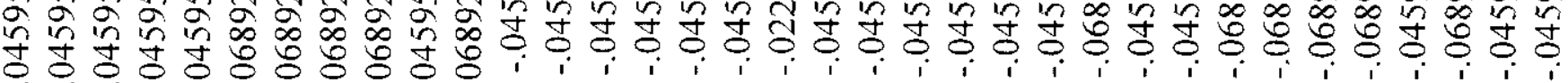
ᄒ́

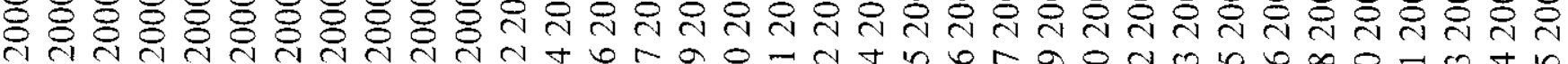

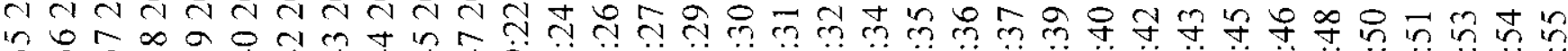

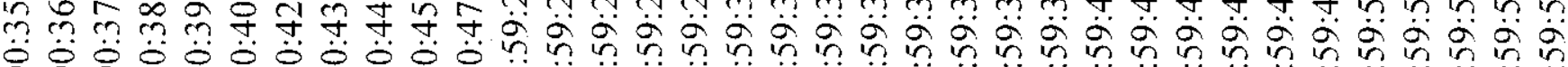

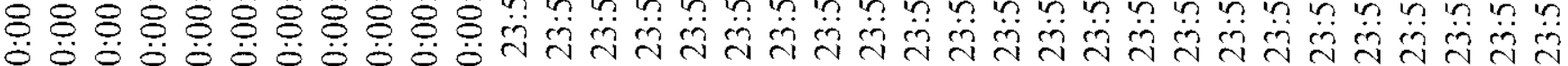

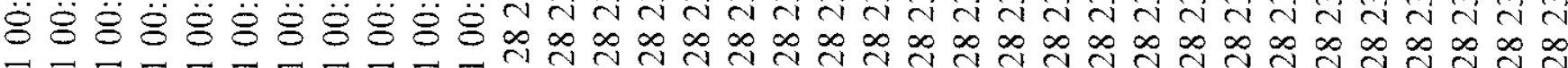

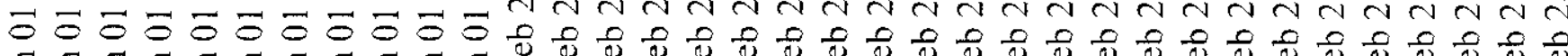

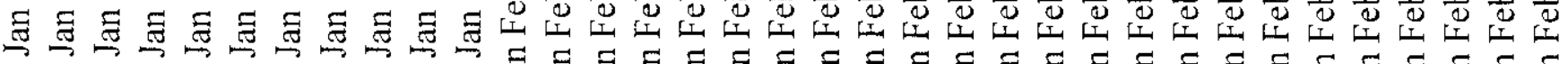

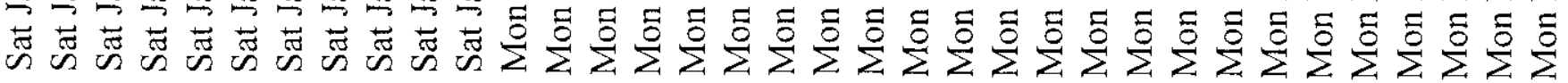




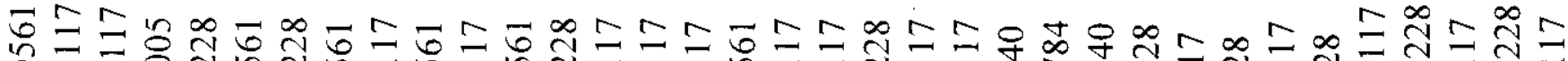

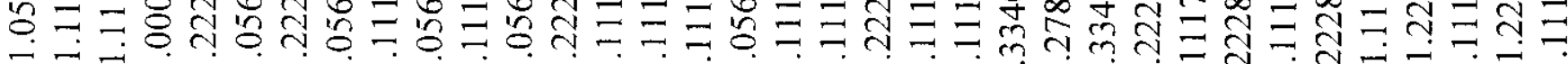

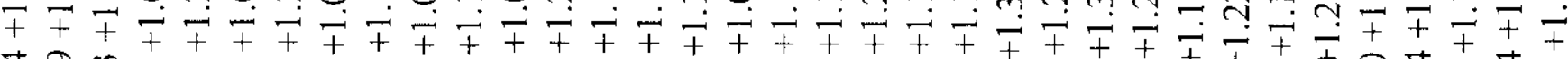

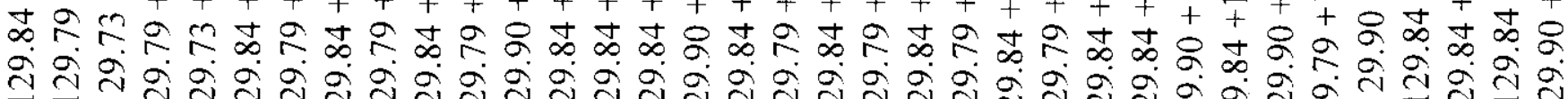

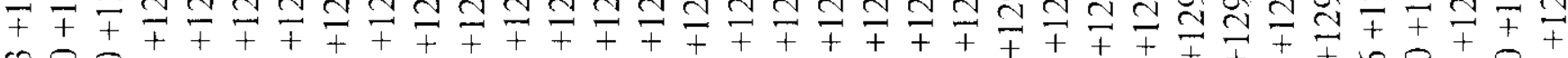

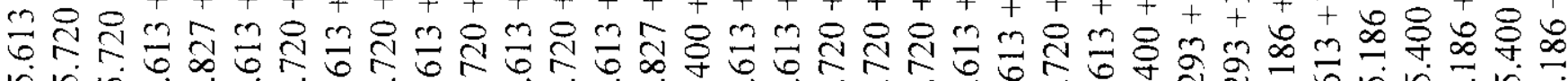

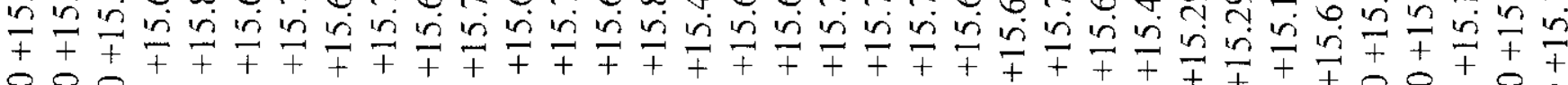

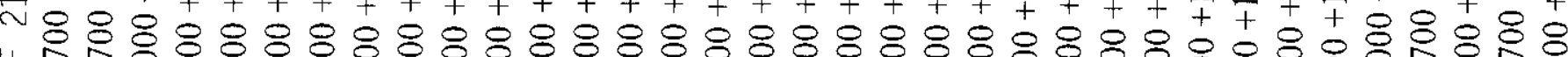

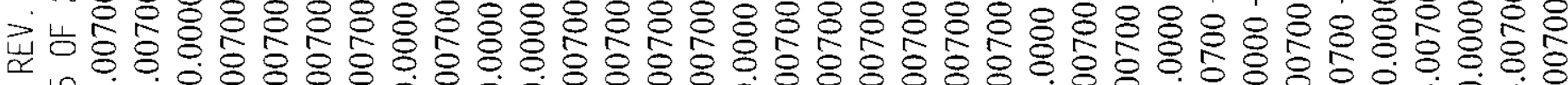

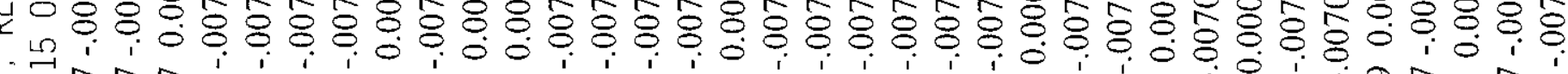
운

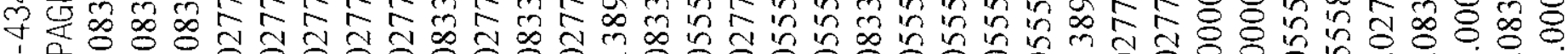

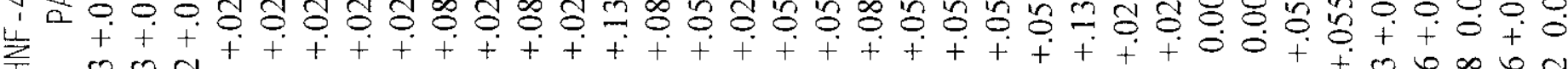
क人 $\infty 00$

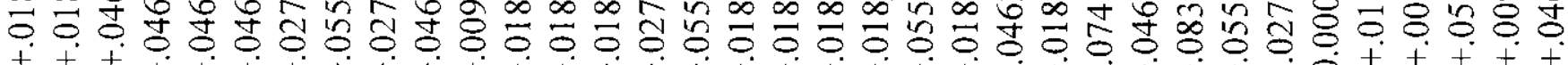

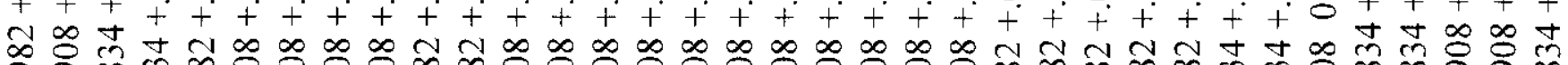
\%

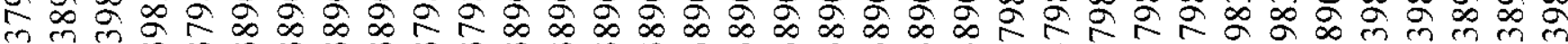

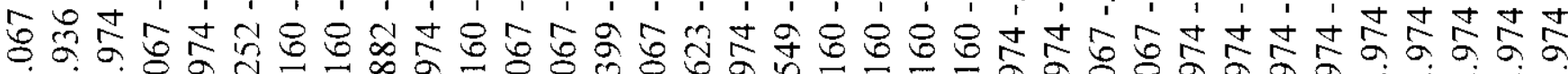
N + +

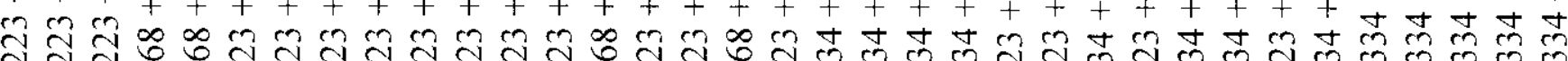

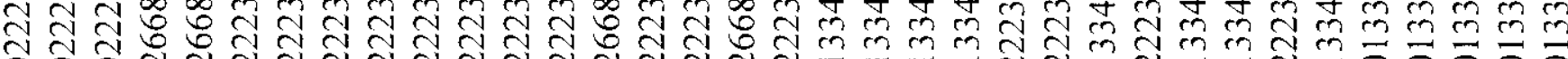

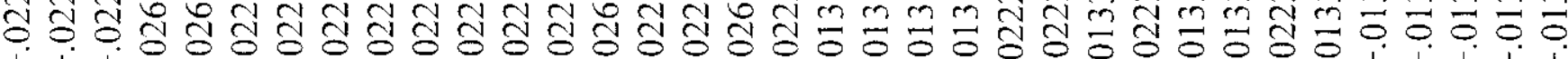

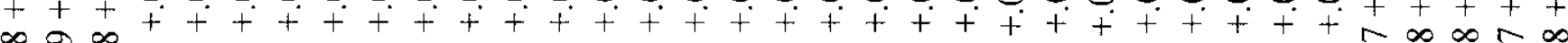

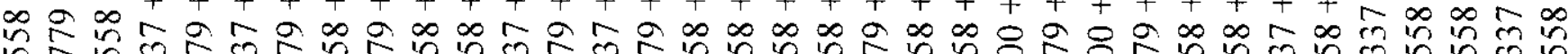

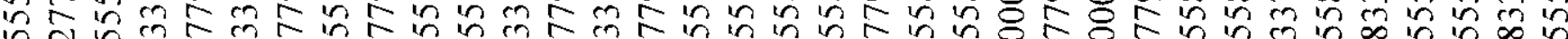

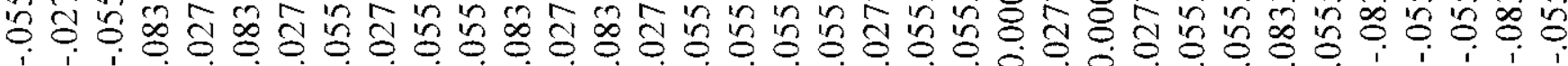

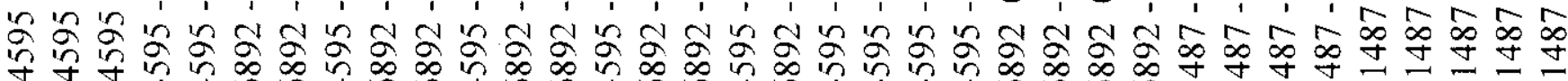

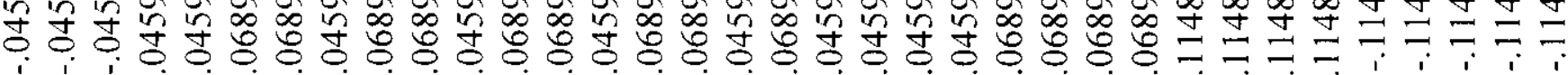

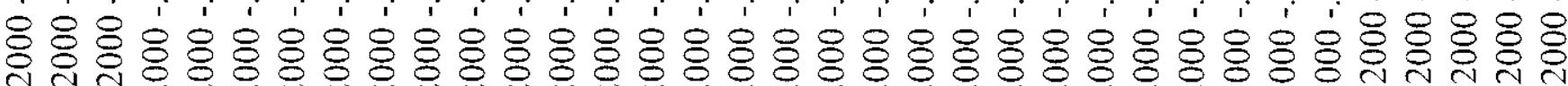
$6 \infty 0$

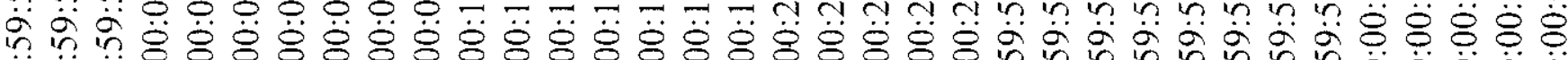

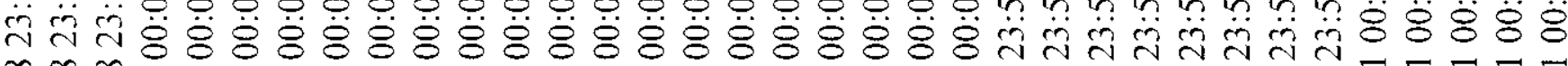
क्र

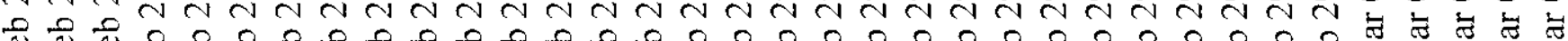

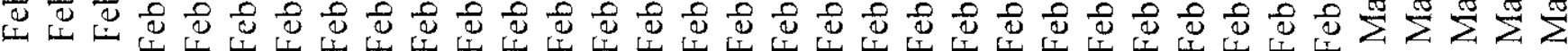

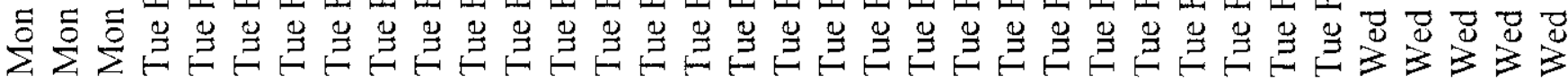




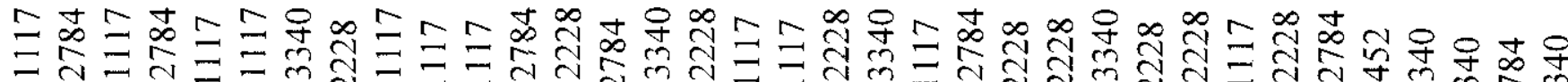

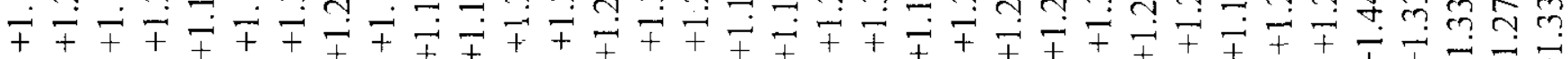

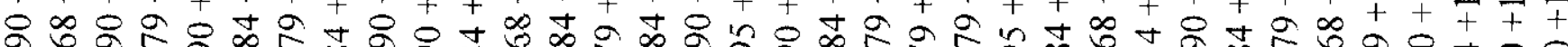

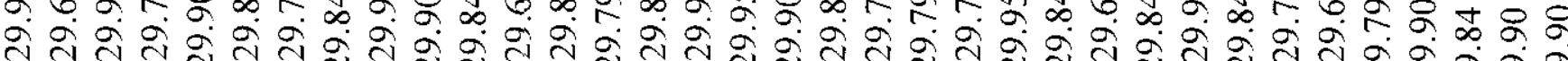

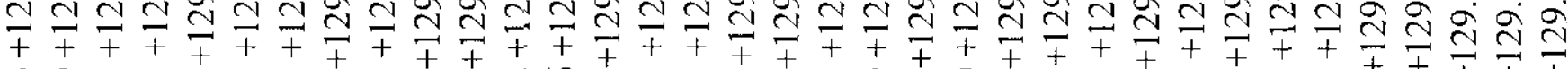
œ

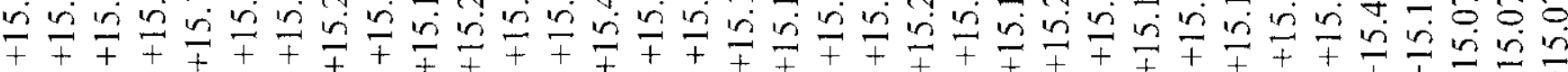

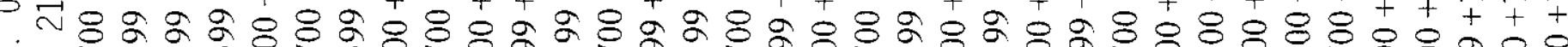
능응m ш 네

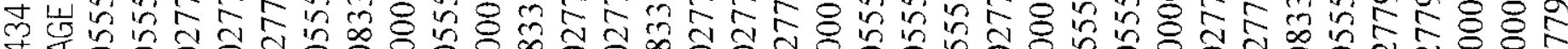
广过。

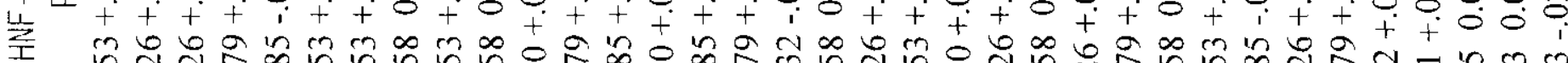

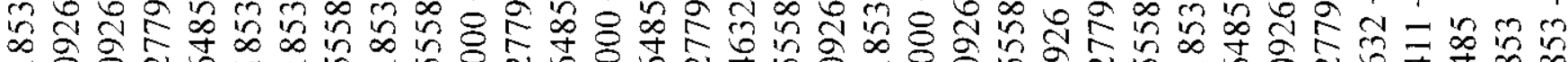

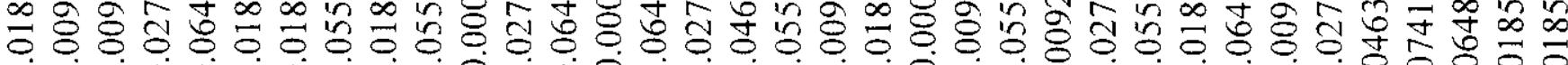

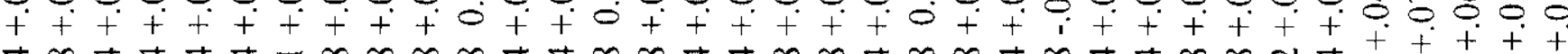

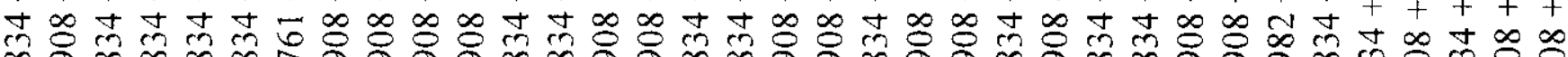

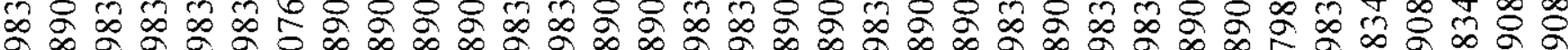

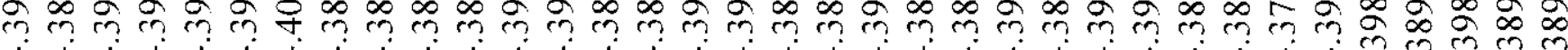

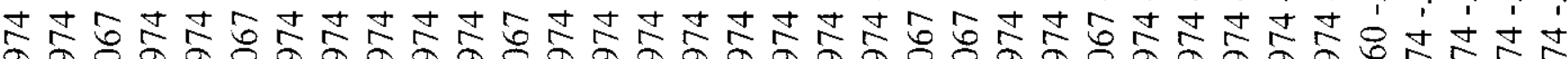
. ปูป

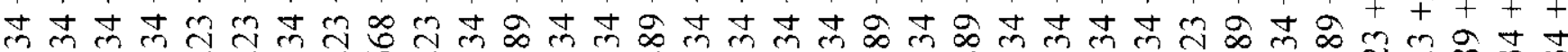
mmm $m$

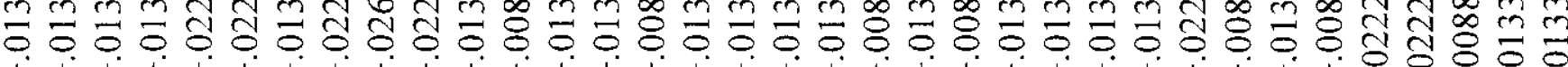

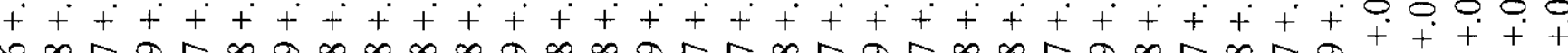

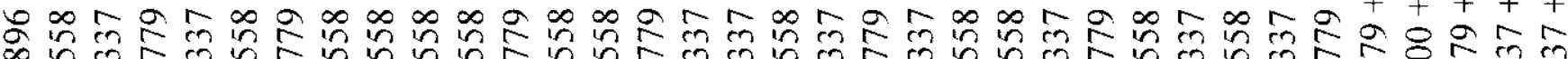
๙

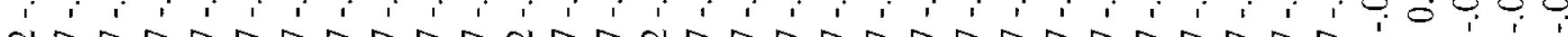

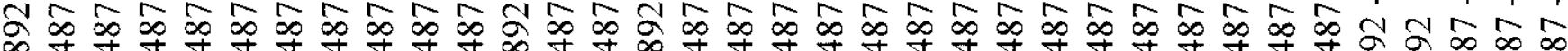

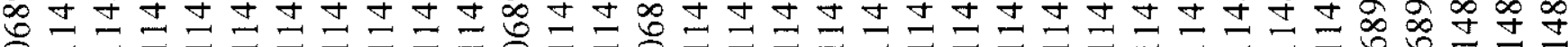

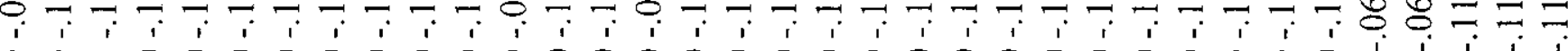

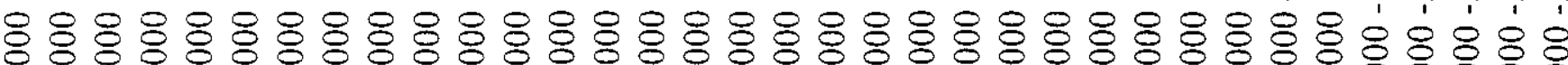

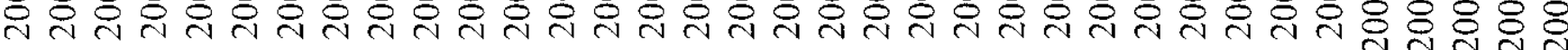

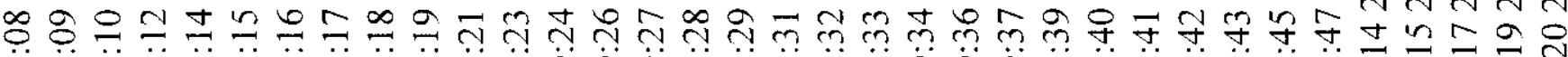
த் த் $\dot{8} \dot{8} \dot{8} \dot{8} \dot{8} \dot{8} \dot{8} \dot{8} \dot{8} \dot{8} \dot{8} \dot{8} \dot{8} \dot{8} \dot{8} \dot{8} \dot{8} \dot{8} \dot{8} \dot{8} \dot{8} \dot{8} \dot{8} \dot{8} \dot{8} \dot{8} \dot{8} \dot{8} \dot{8} \leadsto$ ஸ

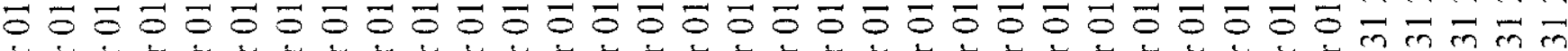

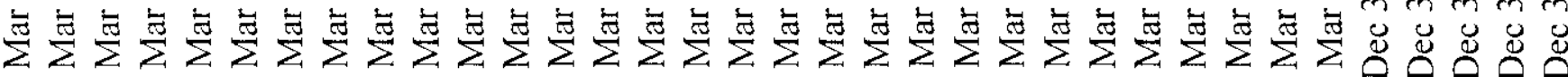

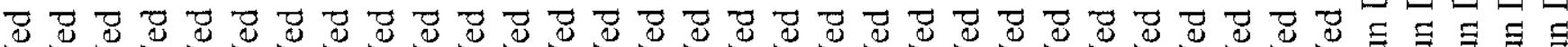

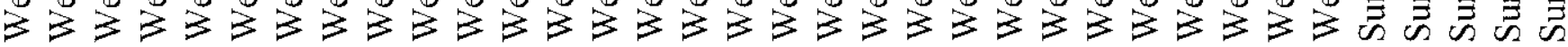




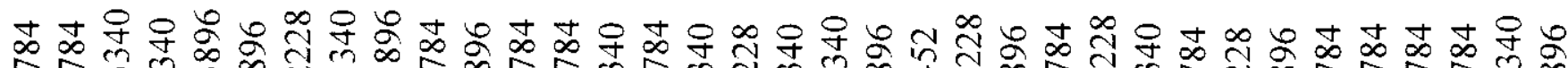

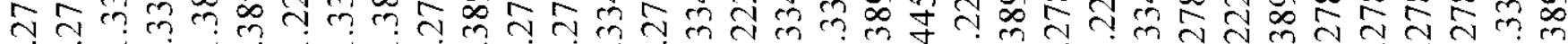

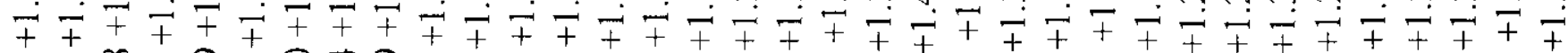

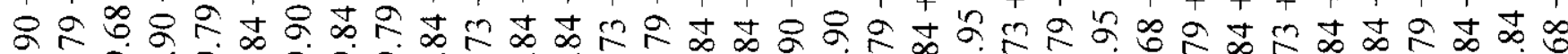

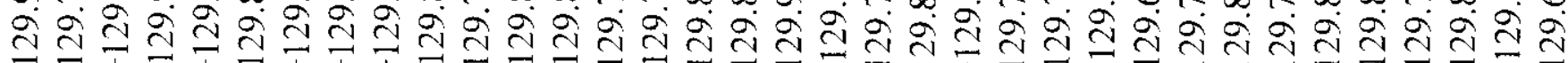

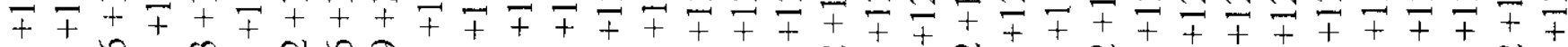

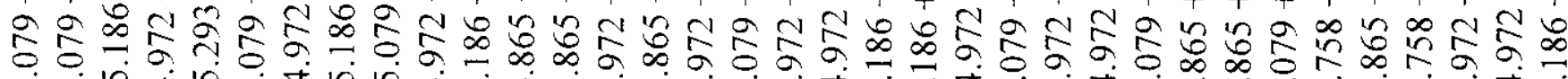

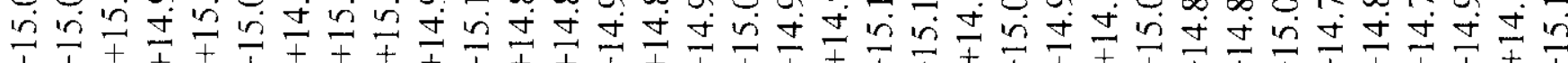
○

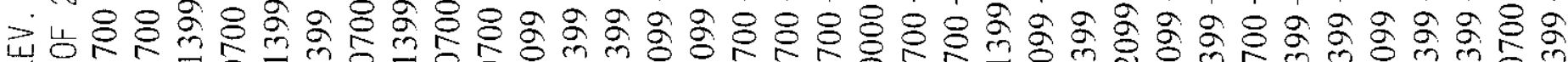
嵌0 二

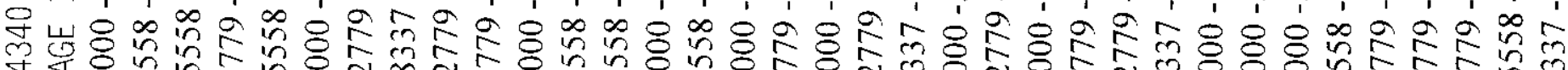

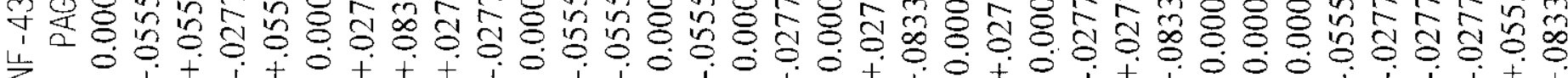

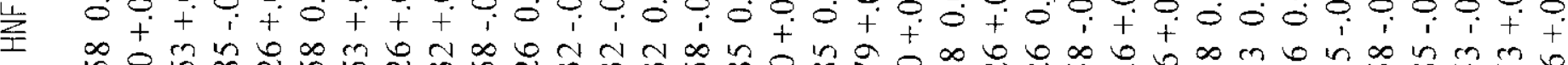

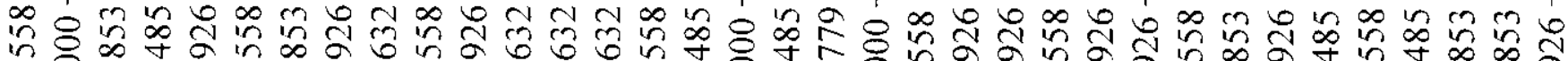

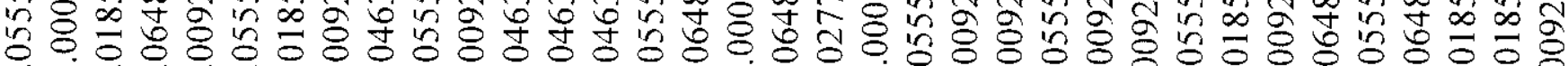
$+0+++++++++++++++0++00+++++i++++++++i$

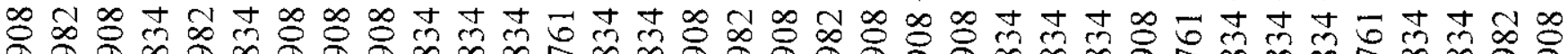

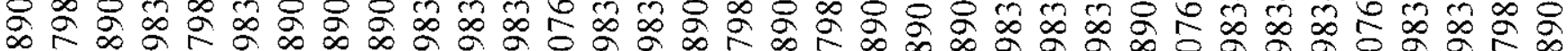
mmmm

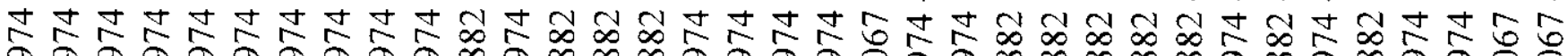
a o o o o a

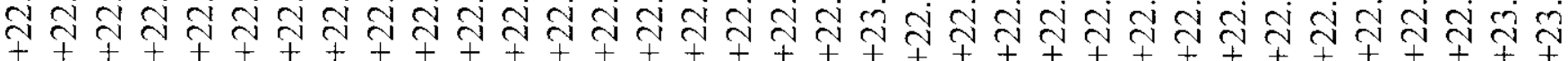

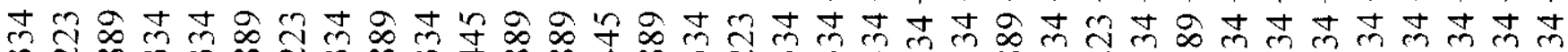

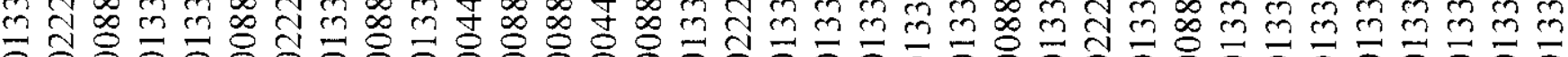

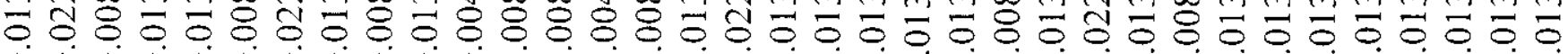
年

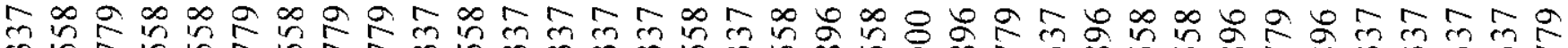

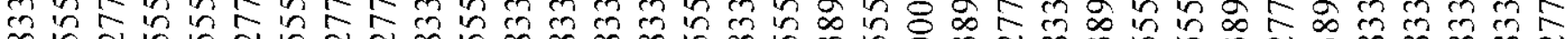

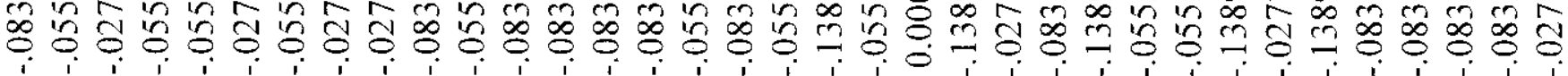

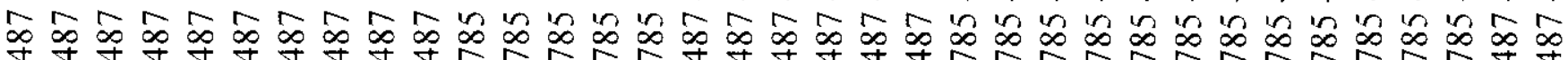

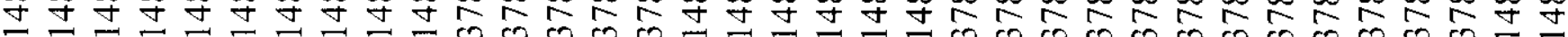
777777777777777777777

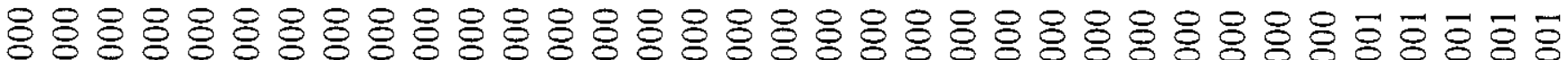

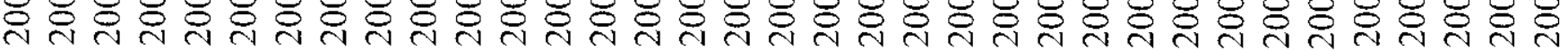

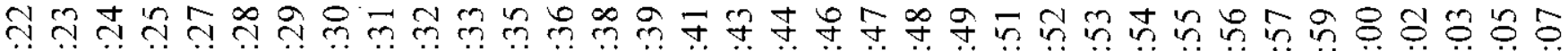

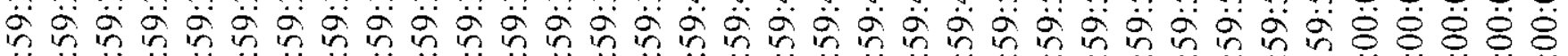

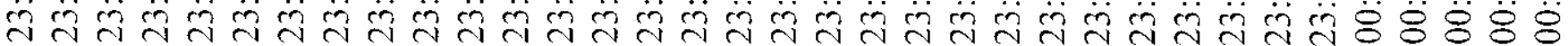

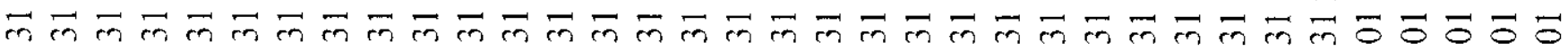

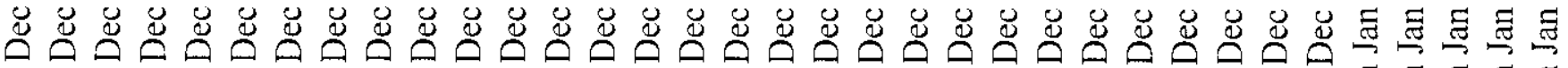

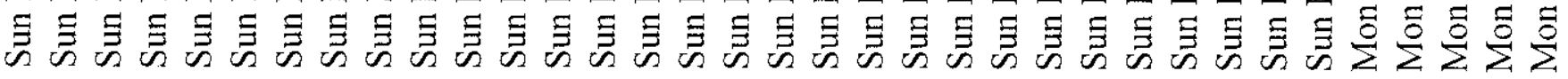




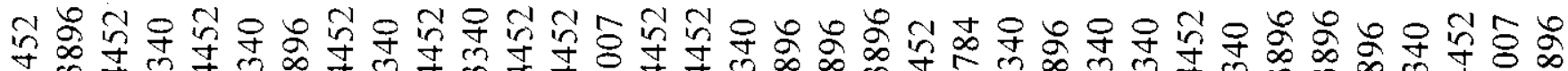

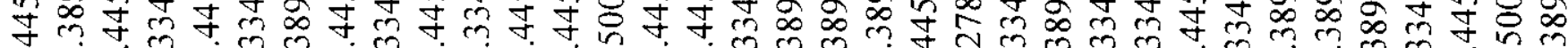

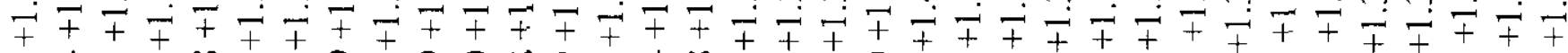

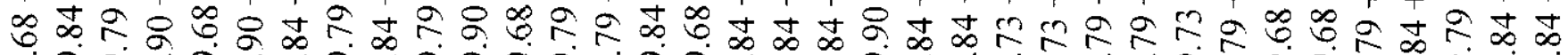
वें बें

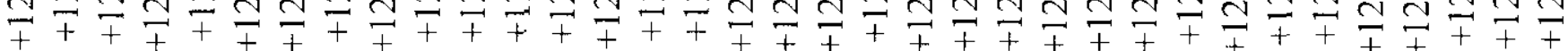

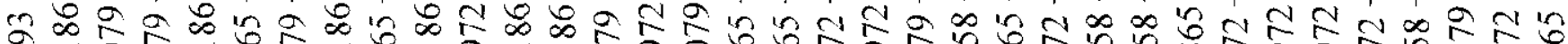
人

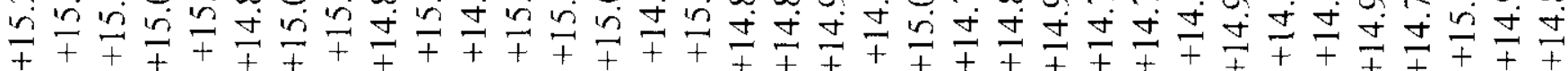

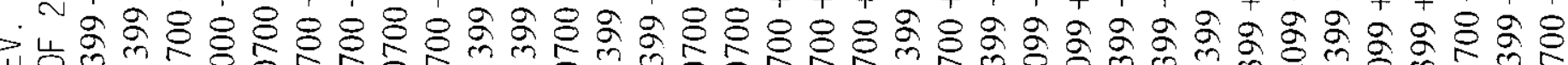
㟧

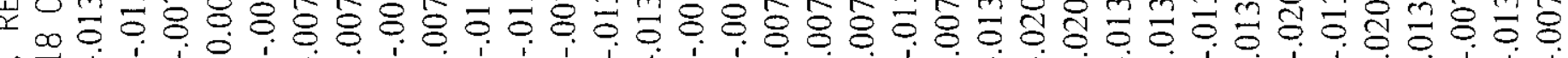

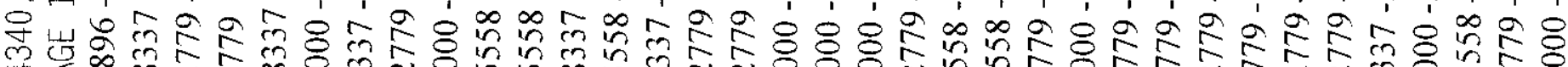

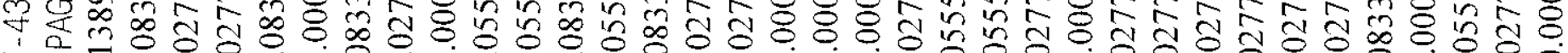

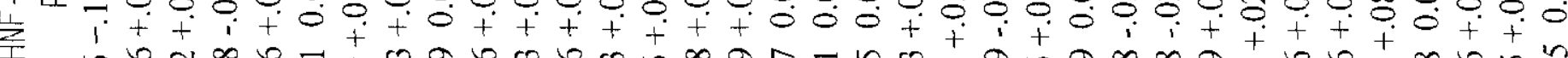

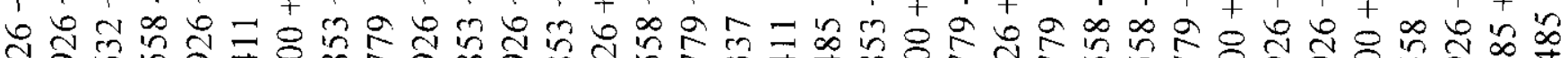

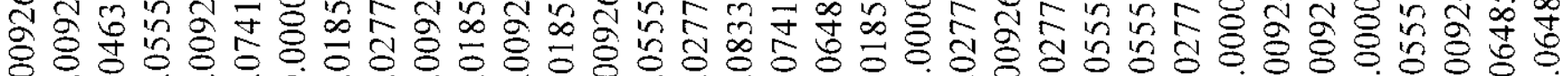

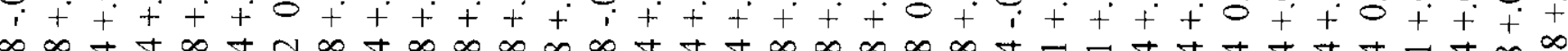

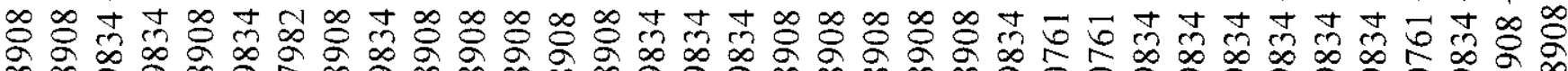

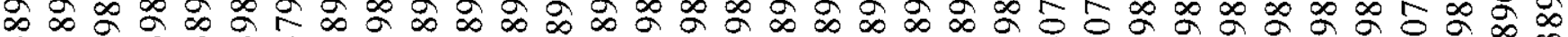
1.

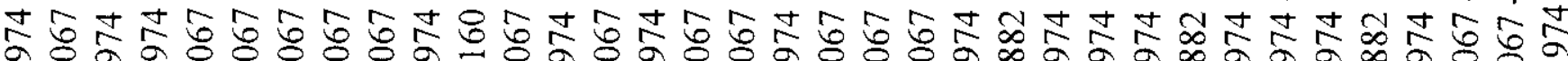

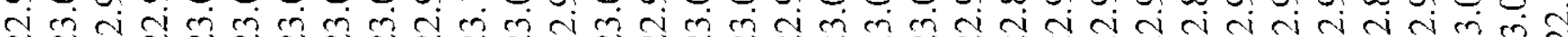

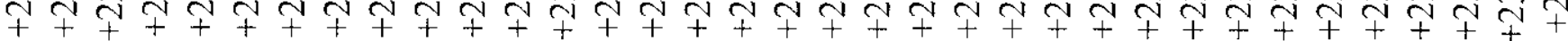
t

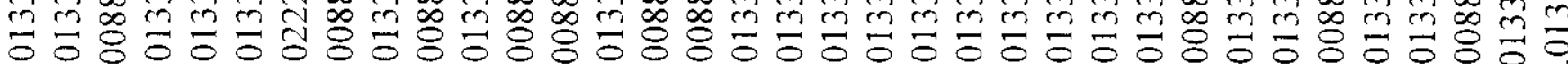

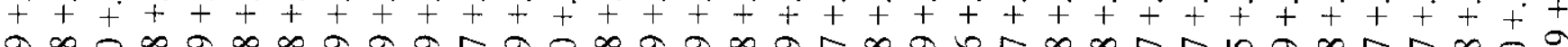

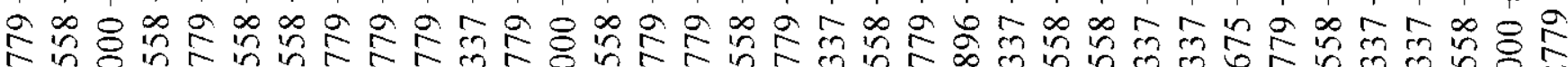

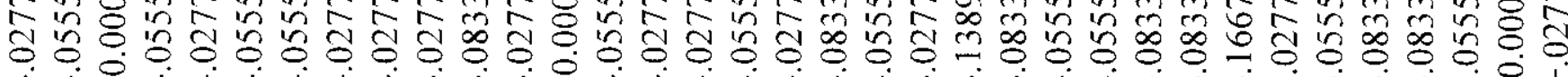

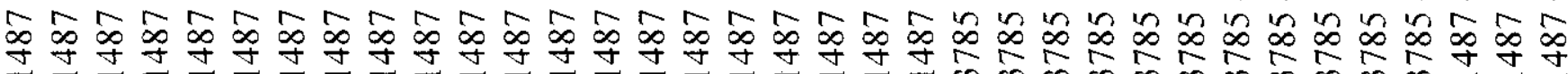

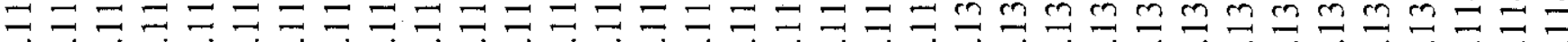

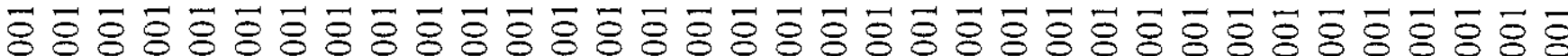

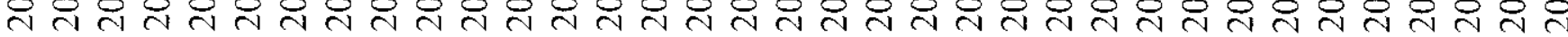

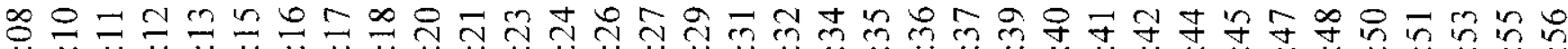

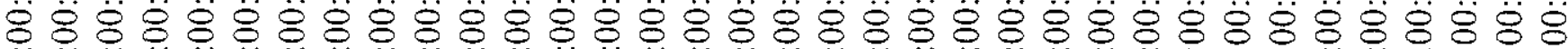

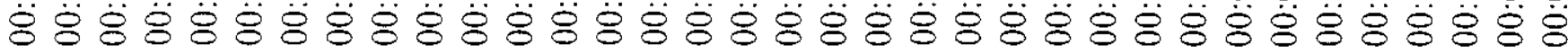

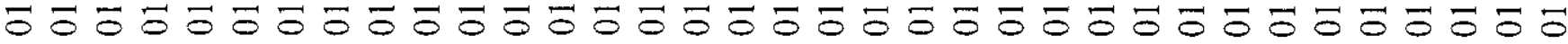

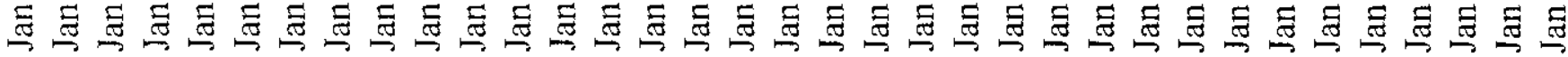

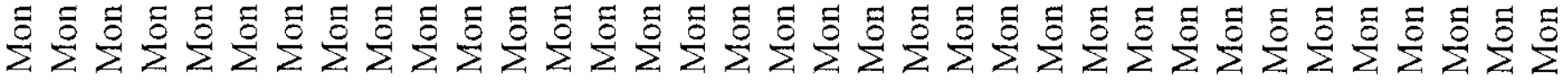




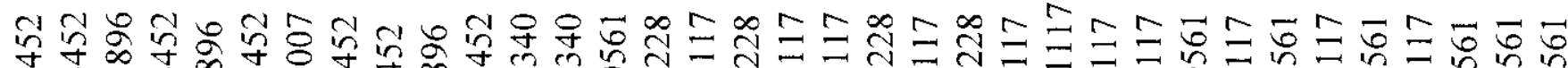

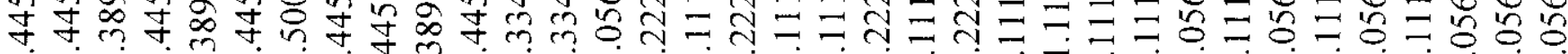

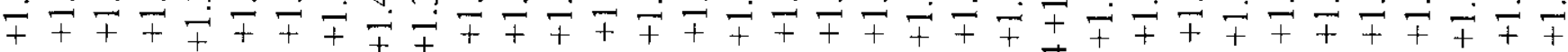

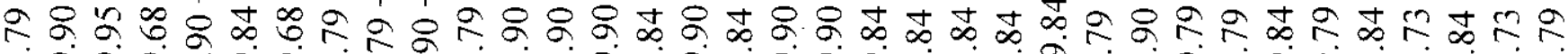

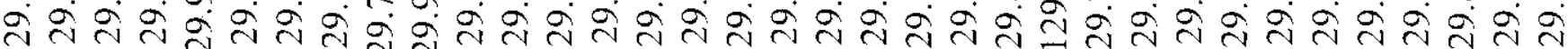

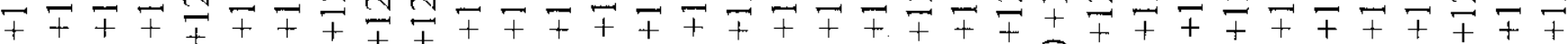

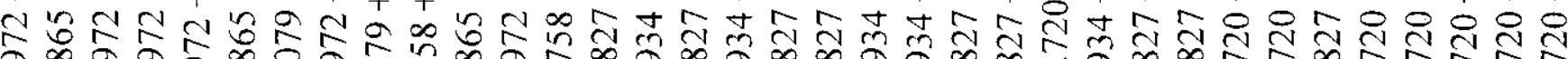

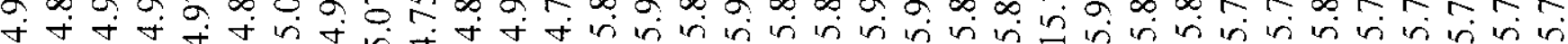

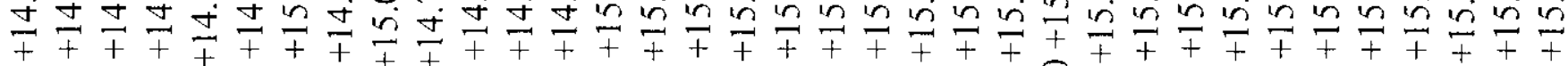

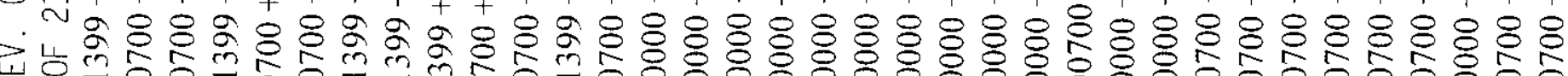

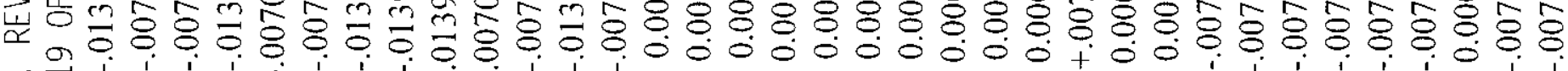

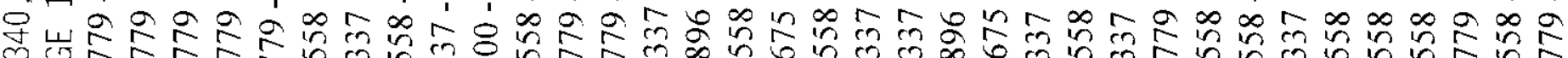

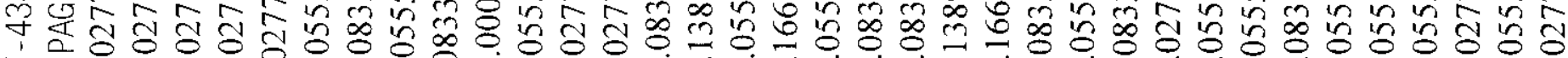
崖 $++++i++++0++++++++++++++++++++++++++$

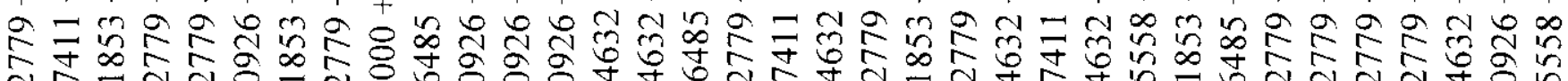

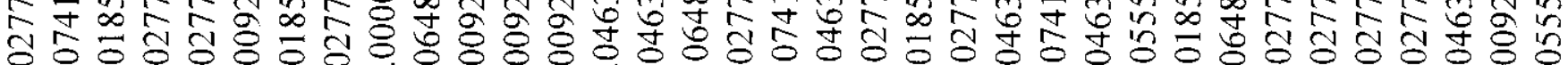
$++++++++0+++++++++++++++++++++++++++$

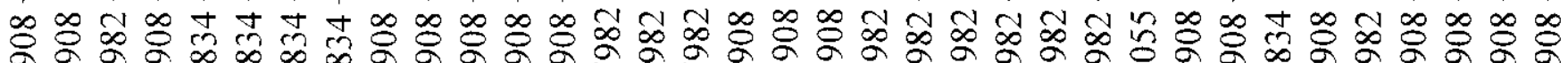

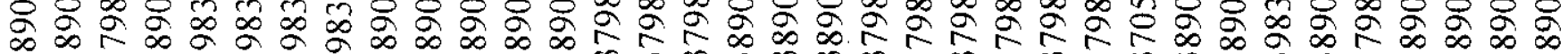
m

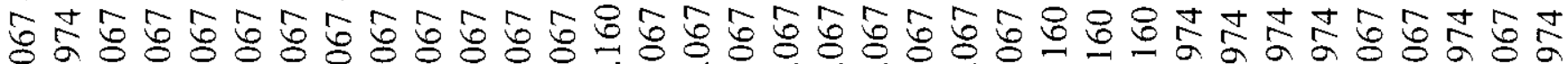

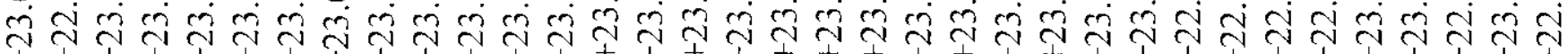

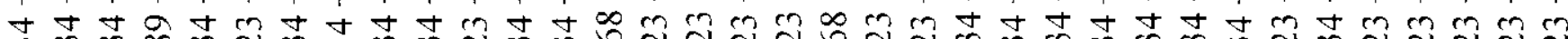
med

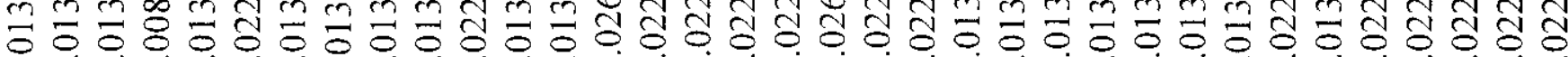

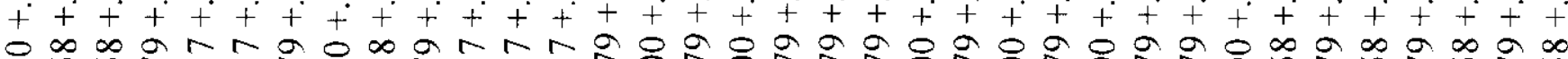

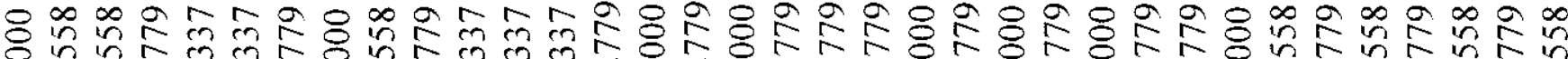
o.

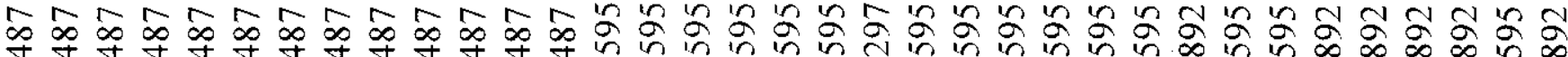

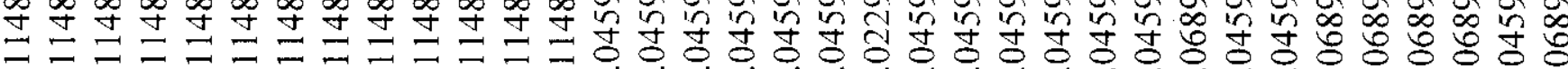

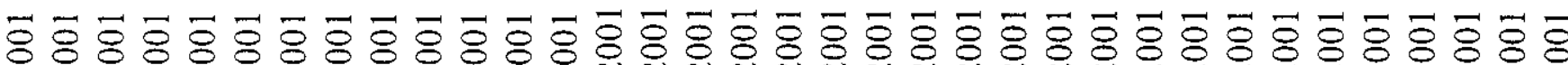

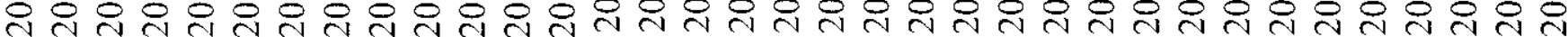

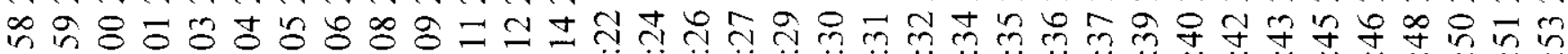

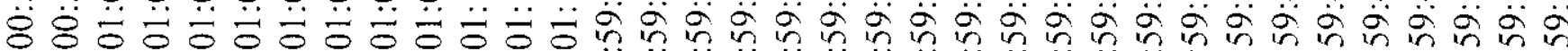

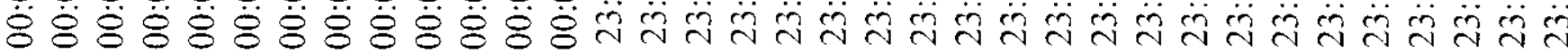

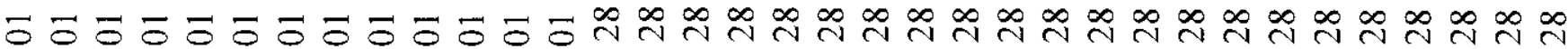

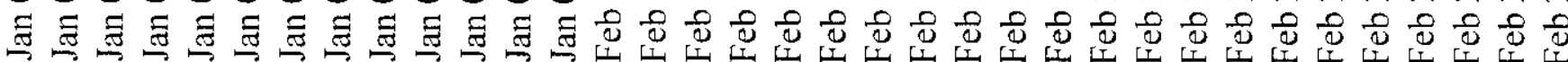
б

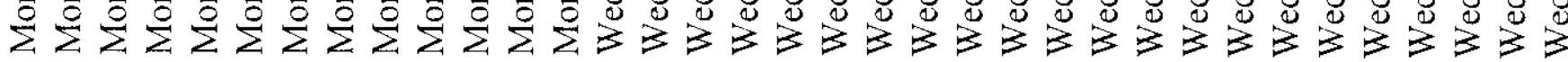




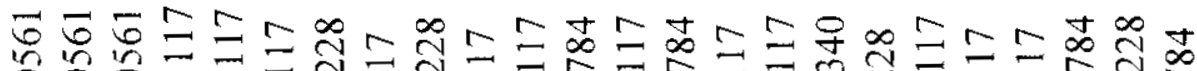
:

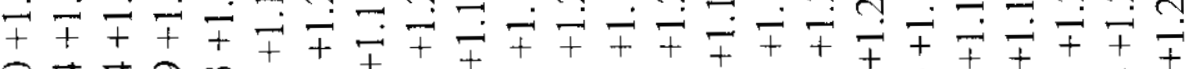

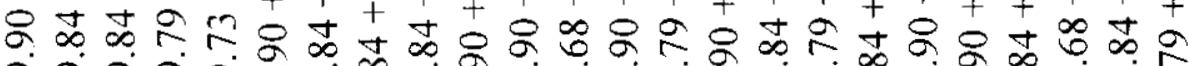
वें

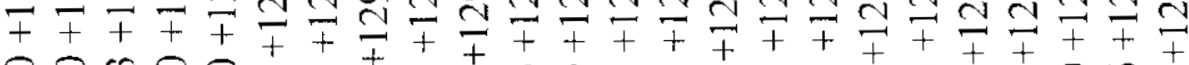

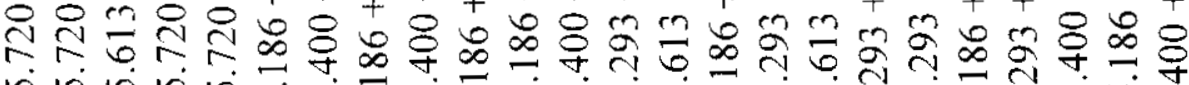

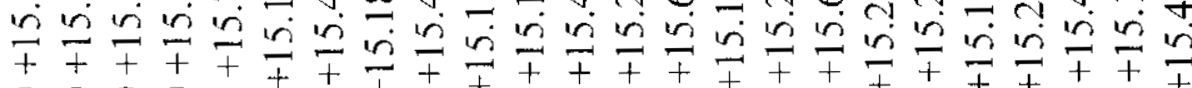

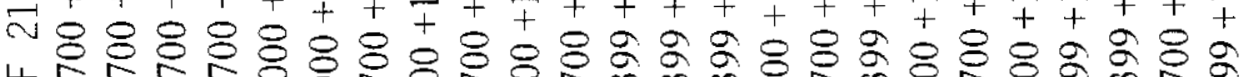

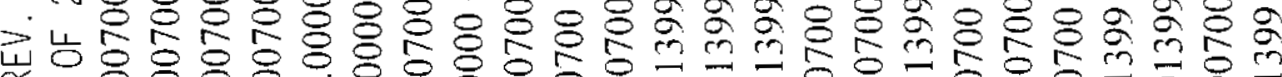
4.

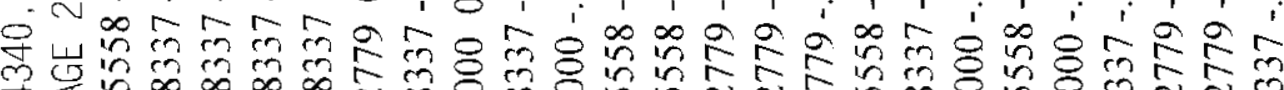

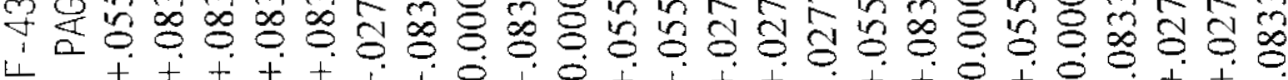
崖 $++++++0+0++++i+0+0+++1$

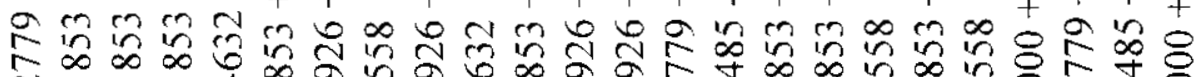

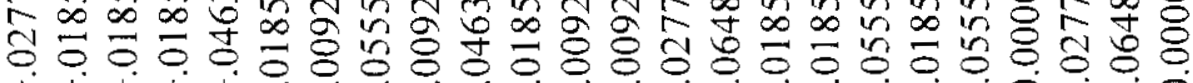
$++++++++++++++++++++0++0$

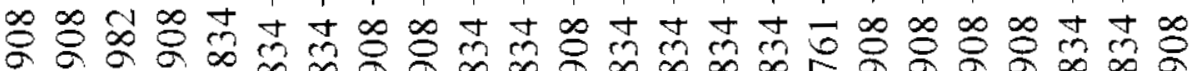
等

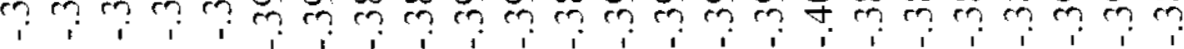

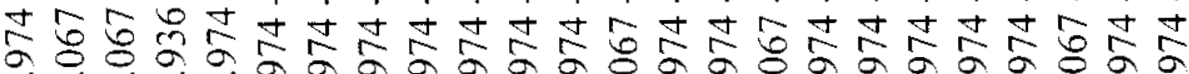

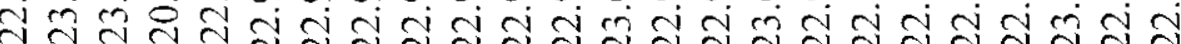

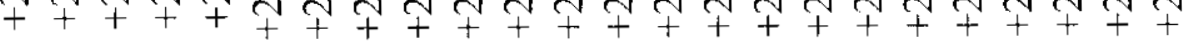

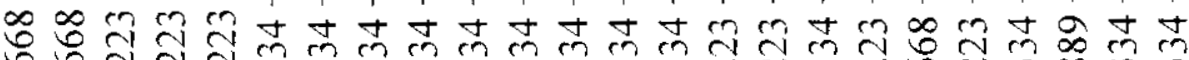
웅 त्र तै तो

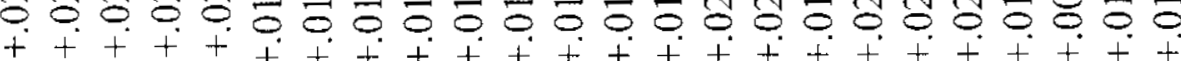

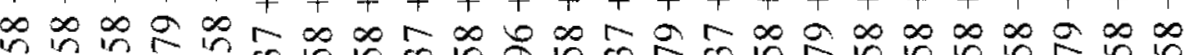

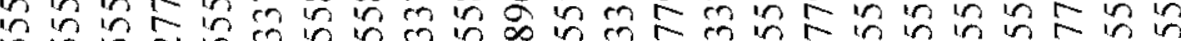

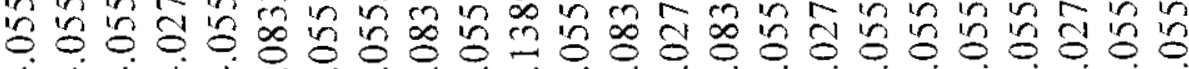

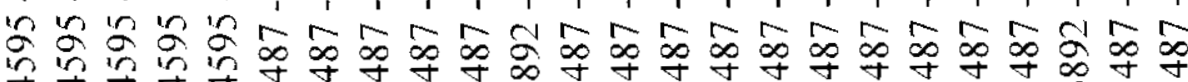

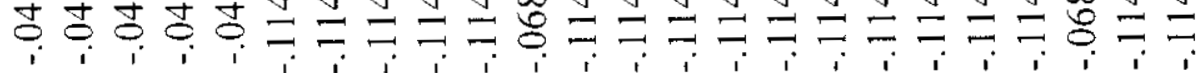

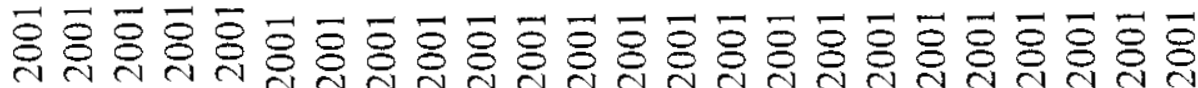
t in

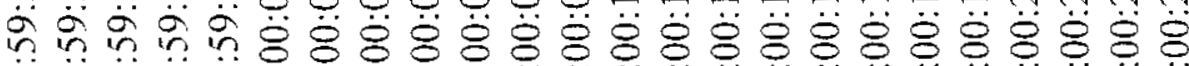

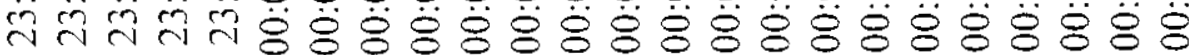
i i i i i 5050505050505005000

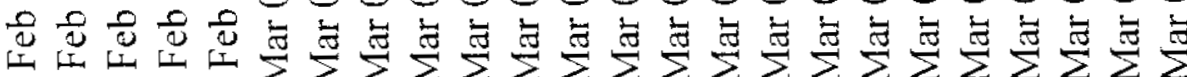

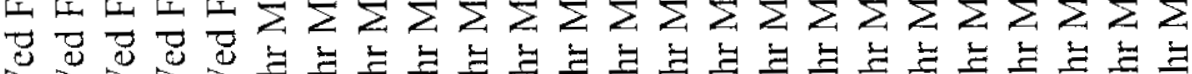

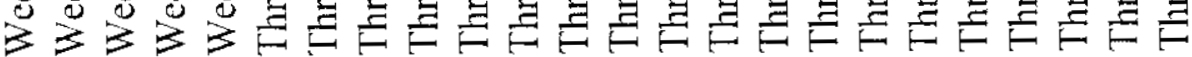




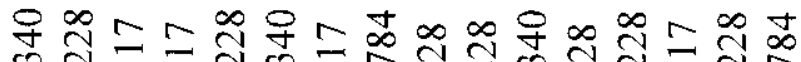
กู่

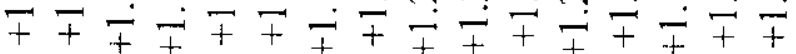

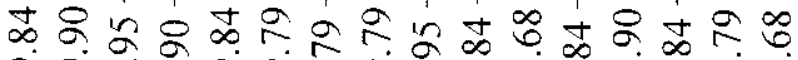

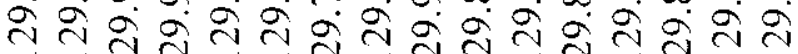

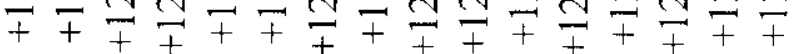

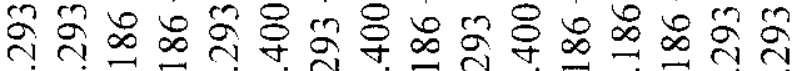

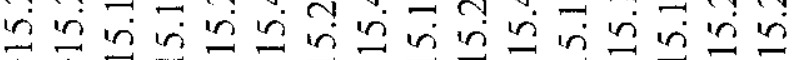

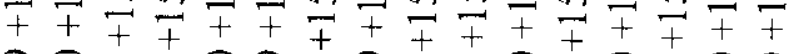

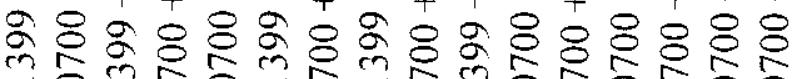

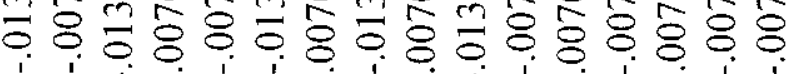

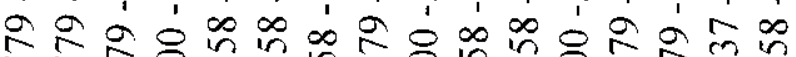

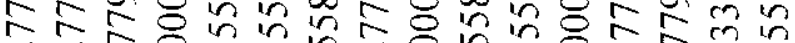

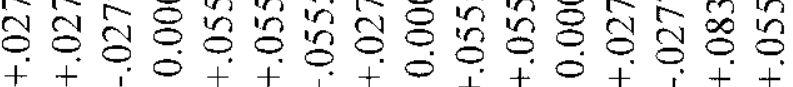

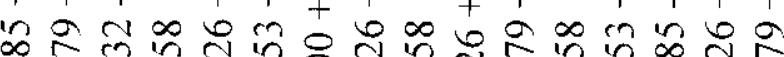
+

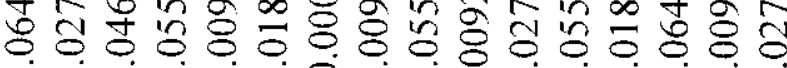
$++++++0++1++++++$ $\infty$ অ্

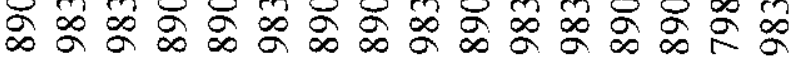
m? m? m? ? m? "? ? ? ? ? ?

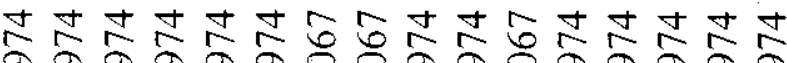
ن

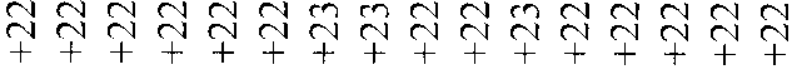

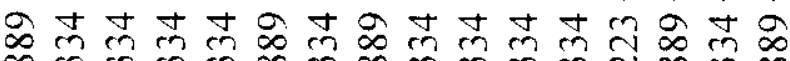
$\infty m m m \infty m \infty m m m_{\infty}^{\infty} m m_{\infty}^{\infty}$

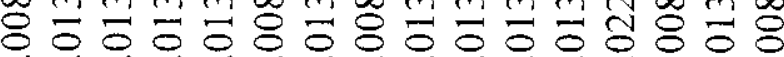

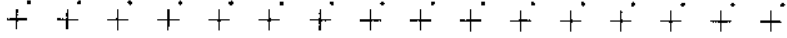
슨

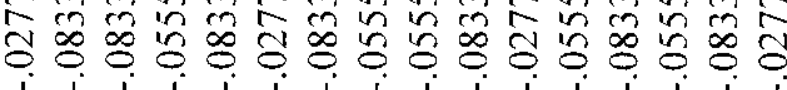
నं

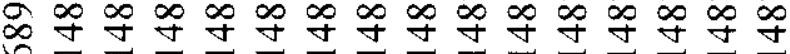

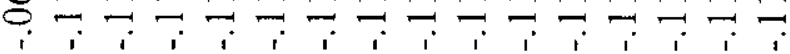

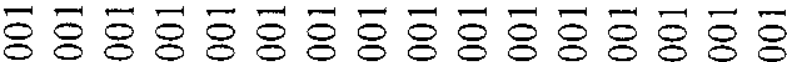

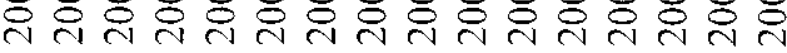
तิ

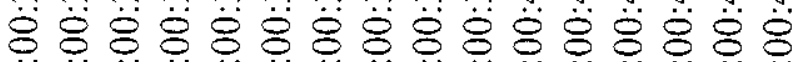
த் $\dot{8} \dot{8} \dot{8} \dot{8} \dot{8} \dot{8} \dot{8} \dot{8} \dot{8} \dot{8} \dot{8} \dot{8} \dot{8}$

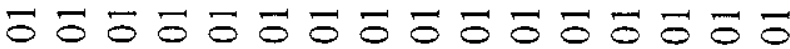

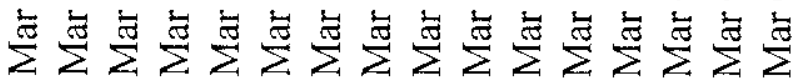

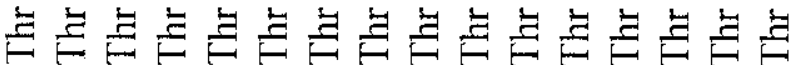

